TI 2014-100/VIII

Tinbergen Institute Discussion Paper

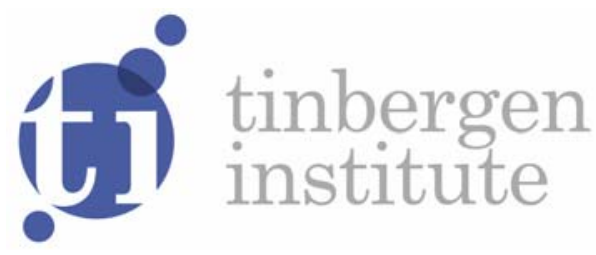

\title{
Carbon Lock-In: The Role of Expectations
}

Gerard van der Meijden'

Sjak Smulders 2

1 Faculty of Economics and Business Administration, VU University Amsterdam, and Tinbergen Institute, The Netherlands;

2 Tilburg University, The Netherlands. 
Tinbergen Institute is the graduate school and research institute in economics of Erasmus University Rotterdam, the University of Amsterdam and VU University Amsterdam.

More TI discussion papers can be downloaded at http://www.tinbergen.nl

Tinbergen Institute has two locations:

Tinbergen Institute Amsterdam

Gustav Mahlerplein 117

1082 MS Amsterdam

The Netherlands

Tel.: +31(0)20525 1600

Tinbergen Institute Rotterdam

Burg. Oudlaan 50

3062 PA Rotterdam

The Netherlands

Tel.: +31(0)10 4088900

Fax: +31(0)10 4089031 


\title{
Carbon Lock-In: The Role of Expectations*
}

\author{
Gerard van der Meijden ${ }^{1}$ \\ Vrije Universiteit Amsterdam, The Netherlands \\ Tinbergen Institute, The Netherlands
}

\author{
Sjak Smulders \\ Tilburg University, The Netherlands \\ CESifo, Germany
}

July 2016

\begin{abstract}
We argue that expectations about future energy use affect the transition from fossil to renewables, because of an interaction between innovation and resource scarcity. The paper presents a model of directed technical change to study this interaction. We find that fossil-saving technical change erodes the incentives to implement renewables. Conversely, the anticipation of a transition to renewables diminishes the incentives to invest in fossil technology. As a result, two equilibria may arise, one with a transition to renewables and with low fossil efficiency, and one without renewables and with high fossil efficiency. Expectations determine which equilibrium arises in equilibrium.
\end{abstract}

JEL codes: O30, Q32, Q42, Q55

Keywords: Directed technical change, energy transition, multiple equilibria

\footnotetext{
*Manuscript received: August 2014; revised: July 2015 and June 2016.

${ }^{1}$ The authors would like to thank Christian Bogmans, Reyer Gerlagh, David Hemous, Jenny Ligthart, Rick van der Ploeg, Francesco Ricci, Luc Rouge, Mark Schopf, Cees Withagen, Aart de Zeeuw, Gijsbert Zwart, participants of conferences and seminars in Amsterdam, Berlin, Fontainebleau, Hamburg, London and Toulouse, and two anonymous referees for their valuable comments. The authors gratefully acknowledge financial support from FP7IDEAS-ERC Grant No. 269788 (GP) and FP7-SSH Grant No. 613420 (GLAMURS). Please address correspondence to: Gerard van der Meijden, Department of Spatial Economics, Vrije Universiteit Amsterdam, De Boelelaan 1105, 1081 HV, Amsterdam, The Netherlands, Phone: +31-20-598-2840, E-mail: g.c.vander.meijden@vu.nl.
} 


\section{Introduction}

For many decades a major engine of growth in the world economy has been the reliable supply of fossil energy resources. Policy makers have been forced, however, to rethink the dominant role of fossil fuels in energy supply, when facing the challenge of combating climate change and the global concern about the sustainability of current living standards. Part of the solution to both the climate change and the sustainability problem may be a phasing out of non-renewable natural resources like fossil fuels and the implementation of backstop technologies that provide renewable substitutes. A more incremental solution would arise from improving resource efficiency and slowing depletion of fossil resources. The question arises how market parties respond to the challenges and which incentives arise over time to invest in resource saving and energy transition. We argue that the energy future of a growing economy is crucially shaped by a two-way interaction between innovation decisions and energy supply decisions. Prospects about future energy generation technologies may affect the time path of fossil fuel supply, but also the pace and direction of technical progress. Conversely, the speed and direction of technical progress are crucial for the transition from fossil fuels to backstop technologies. This two-way interaction or complementarity between innovation and resource supply may create multiple equilibria and self-fulfilling prophecies.

Since our question concerns the dynamics of energy use and technology in a growing economy, we naturally frame our analysis in a growth model with natural resources and endogenous technical change. Our starting point is the Dasgupta-Heal-Solow-Stiglitz (DHSS) model $^{2}$ in which a scarce non-renewable resource (fossil) is an essential input in production. We allow fossil energy to be replaced by renewable energy that can be generated at a constant cost, the so-called backstop technology (cf. Nordhaus, 1973). As is well known, in the DHSS model growth cannot be sustained unless resource-augmenting technical change offsets the negative growth impact of declining availability of the non-renewable resources. At the same time, labor-augmenting technical change fuels growth and boosts the demand for energy. Energy demand thus results from the balance between two types of innovation, resource-augmenting and labor-augmenting technical change. We incorporate both types in our analysis and allow profit incentives to guide innovators how much and in which direction to innovate. Thus, we merge the DHSS model with a model of directed technical change. ${ }^{3}$

Our main finding is that the replacement of fossil resources might require a coordination of

\footnotetext{
${ }^{2}$ See Dasgupta and Heal (1974), Solow (1974a,b), Stiglitz (1974a,b), Benchekroun and Withagen (2011), and Van der Ploeg and Withagen (2014).

${ }^{3}$ The literature on induced innovations was introduced by Hicks (1932) and more recently formalized in the directed technical change models of Acemoglu (1998; 2002; 2003) and Kiley (1999). We choose for investment in knowledge instead of in physical capital to orient our analysis towards the long run, when technical change rather than capital accumulation is the determinant of output growth.
} 
expectations, because a coordination failure may arise due to the existence of a strategic complementarity in investment in resource conservation and investment in resource-augmenting technologies. High investment in resource conservation stimulates investment in resourceaugmenting technologies and vice versa so that if atomistic investors make investment decisions on resource conservation or innovation, their return depends positively on the investment decisions of other investors on the complementary investment, innovation or conservation, respectively (cf. Cooper and John, 1988). Intuitively, the reasoning is as follows. If the costs of generating energy with the backstop technology are sufficiently low, it is a viable alternative to fossil fuels in the long run. However, resource conservation together with investment in resource-saving technical change can make fossil effectively cheaper to use than the backstop. Whether in equilibrium fossil is phased out or not then depends on the expectations of fossil suppliers and innovators. A self-fulfilling prophecy arises since when it is expected that the backstop will be implemented, the market for the resource and for resource-saving inventions will be small implying that conservation and innovation incentives will be eroded; this makes the backstop relatively more attractive and thus justifies the expectation that the backstop will be implemented. Conversely, when no future backstop deployment is expected, resource conservation and resource-saving technical change become more profitable, thus making the resource indeed relatively more attractive in the long run. Only when the backstop cost is below a certain threshold, it will always be deployed in the long run.

Our results imply that it might be hard to steer the economy away from the current dependence on fossil fuels because it is 'locked into fossil' (cf. Unruh, 2000): mutual welfare gains from an economy-wide change in investment decisions may not be realized, because no individual investor has an incentive to deviate from the initial market equilibrium (cf. Cooper and John, 1988).

Lock-in and multiple equilibria are studied in the literature in several settings. ${ }^{4}$ Cooper and John (1988) showed that a necessary condition for obtaining multiple equilibria in a static setting is the existence of strategic complementarities, which are said to arise "when the optimal strategy of an agent depends positively upon the strategies of the other agents" (Cooper and John, 1988, p. 441). Murphy et al. (1989) use a model with strategic complementarities between industrializing sectors to show that self-fulfilling expectations may exist: the willingness of firms to invest depends on their expectation that other firms invest, because the future market size that a single firm faces depends on the investment by the other firms. In the growth literature the strategic complementarities are between different capital stocks. In Chamley (1993) and Benhabib and Farmer (1994), a strategic complementarity between the stocks of

\footnotetext{
${ }^{4}$ Arthur (1989) and David (1985) introduced the notion of lock-in into economics.
} 
physical and human capital may create a multiplicity of equilibria (or, in their terminology: 'indeterminacy') in Uzawa-Lucas or Romer type of endogenous growth models (cf. Uzawa, 1965; Romer, 1986; Lucas, 1988). Benhabib and Perli (1994) show that the traditional distinction between physical capital and human capital is not necessary: even with only human capital self-fulfilling prophecies can arise as long as the return to investment in an individual's private human capital stock depends on the human capital investments of others through an aggregate capital stock externality. Benhabib and Perli (1994) make a distinction between local and global indeterminacy as a reason for the existence of multiple equilibria. Local indeterminacy occurs if there exist multiple transitional paths towards a certain balanced growth path, whereas global indeterminacy arises from the existence of multiple balanced growth paths that each can be approached by identically endowed economies with identical initial conditions. Recently, Cozzi $(2005,2007)$ has explored the role of multiple equilibria in a Schumpeterian quality ladders model. He shows that the number of sectors in which R\&D takes place may depend on selffulfilling expectations. Our study complements the literature on multiple equilibria by showing that the existence of global indeterminacy may have important consequences for the transition from fossil fuels to renewable energy sources in a model with directed technical change.

While strategic complementarity is a necessary condition for self-fulfilling prophecies, the seminal paper by Krugman (1991) shows that initial conditions, represented by the initial value of a stock variable, matter as well. The role of 'history' can dominate that of 'expectations' for selecting one of the multiple equilibria. In his model, the historically determined costs advantage of one of the two production sectors must be small enough to make investment into the other sector an equilibrium. Krugman's model features a single state variable and a single non-predetermined variable and can be reduced to an insightful phase diagram with multiple adjustment paths to the two steady states. ${ }^{5}$ Our model is more complex due to multiple state and non-predetermined variables and due to regime changes, but our analysis of the role of history versus expectations relies heavily on Krugman's.

In the context of energy use, there are only a few other studies in which multiple equilibria are discussed. Acemoglu et al. (2012) construct a model with a fossil and a renewables steady state to study lock-in that arises from initial conditions or 'history', viz. innovation in pollutionintensive sectors in the past. Our analysis is complementary to theirs in that we emphasize lock-in that arises from expectations rather than history. Moreover, we adopt a different view of technical change in which society has to choose between incremental change that cannot make scarce resource inputs redundant (because of poor substitution) and radical change in

\footnotetext{
${ }^{5}$ Technically, Krugman (1991) shows that in his model the existence of a role for expectations in selecting the equilibrium requires complex roots around the internal steady state. However, this result depends on the linearity of his system. Matsuyama (1991) shows that in a more general model expectations may select the equilibrium also in case of real roots.
} 
the form of the transition to the backstop. Also in the context of energy use, Schmidt and Marschinski (2009) construct a static, partial equilibrium model to show that due to a strategic complementarity between investment in technology and production capacity, two equilibria can coexist: one with a low and one with a high supply of renewable energy. They use their model to study the possibility of a 'technological breakthrough' in the renewable energy sector. Cheikbossian and Ricci (2013) consider a game between a resource owner and an R\&D firm and show that depending on expectations one out of two equilibria is selected, one with high $R \& D$ and slow depletion, and one with low R\&D and high depletion. Their two-period framework cannot explicitly address the link to economic growth and ignores the possibility of a radical technology change in the form of a backstop, which is the focus of our study.

Directed technical change has been studied in the context of energy scarcity in several studies, with Smulders and de Nooij (2003) as an early example. A key question in this literature concerns the role of resource-augmenting technical change relative to other types of technical change. With resource inputs growing at a lower rate than other inputs, resource-augmenting technical change dominates along a balanced growth path, provided that substitution possibilities are poor, as shown in e.g. André and Smulders (2014). With good substitution, however, resources are not essential for growth and growth can be sustained without technical change in the resource sector, as in Acemoglu et al. (2012). In the model of Di Maria and Valente (2008), in which a non-renewable resource and physical capital are both essential for production, there may be capital-augmenting technical progress in the short run, but technical change will be purely resource-augmenting along any balanced growth path. Pittel and Bretschger (2010) find that technical change is biased towards the resource-intensive sector at the balanced growth equilibrium of their model economy in which sectors are heterogeneous with respect to the intensity of natural resource use. We complement these studies by allowing for a regime shift in energy usage after which the value of accumulated knowledge in the resource sector vanishes.

The remainder of the paper is structured as follows. Section 2 describes the model. Section 3 discusses the dynamics of the model and the energy transition. Section 4 provides a numerical analysis to quantify the results. Section 5 performs a welfare analysis, compares the decentralized equilibrium with the social optimum, and extends the model by including damages from carbon emissions. Finally, Section 6 concludes.

\section{The model}

The model describes a closed economy in which final output is produced with labor and energy services according to a constant elasticity of substitution (CES) production function. In line with the empirical evidence in Koetse et al. (2008) and Van der Werf (2008), energy and man- 
made factors of production are poor substitutes - the elasticity of substitution is smaller than unity. Technical progress is driven by labor allocated to $R \& D$, which is undertaken by firms to improve the quality of their products, as in Acemoglu (1998). As a result, there are two types of technical change in the model: labor-augmenting and resource-augmenting technical change. Although the model has three state variables, we can analyze the dynamics and regime shifts by using two-dimensional phase diagrams.

\subsection{Firms}

Final output $Y$ is produced using labor services $Y_{L}$ and energy services $Y_{E}$ according to the following CES specification: ${ }^{6}$

$$
Y=\left[\gamma Y_{L}^{\frac{\sigma-1}{\sigma}}+(1-\gamma) Y_{E}^{\frac{\sigma-1}{\sigma}}\right]^{\frac{\sigma}{\sigma-1}}
$$

where $\gamma$ regulates the relative productivity of the inputs and $\sigma \in(0,1)$ denotes the elasticity of substitution between labor and energy services. Profit maximization under perfect competition gives rise to the following factor demand functions:

$$
\begin{array}{r}
p_{Y} \gamma Y_{L}^{-\frac{1}{\sigma}}\left(\gamma Y_{L}^{\frac{\sigma-1}{\sigma}}+(1-\gamma) Y_{E}^{\frac{\sigma-1}{\sigma}}\right)^{\frac{1}{\sigma-1}}=p_{Y L}, \\
p_{Y}(1-\gamma) Y_{E}^{-\frac{1}{\sigma}}\left(\gamma Y_{L}^{\frac{\sigma-1}{\sigma}}+(1-\gamma) Y_{E}^{\frac{\sigma-1}{\sigma}}\right)^{\frac{1}{\sigma-1}}=p_{Y E},
\end{array}
$$

where $p_{Y}, p_{Y L}$, and $p_{Y E}$ are the prices of output, labor services, and energy services, respectively.

Energy is derived from resource services $Y_{R}$ or generated by the backstop technology sector $Y_{H}$. Both energy sources are perfect substitutes. ${ }^{7}$ The generation of energy by the backstop technology requires the final good as input. Hence, the energy sector is described by

$$
\begin{aligned}
& Y_{E}=Y_{R}+Y_{H}, \\
& Y_{H}=\eta H,
\end{aligned}
$$

where $\eta>1$ is a productivity parameter and $H$ denotes the input of the final good. Labor and resource services are produced according to the following Cobb-Douglas specification:

$$
Y_{i}=M_{i}^{\beta} \int_{0}^{1} q_{i k} x_{i k}^{1-\beta} d k
$$

\footnotetext{
${ }^{6}$ The time argument $t$ is omitted if there is no possibility of confusion.

${ }^{7}$ We study the extreme case of perfect substitutability between the resource and the backstop for simplicity. In a similar context, Van der Meijden (2013, pp. 223-227) shows that results remain unaffected qualitatively as long as the elasticity of substitution between the resource and the backstop is high enough.
} 
where $i=\{L, R\}$, and $M_{L}=L$ and $M_{R}=R$ denote the inputs of labor and the resource, respectively. The amount and quality of intermediate good variety $k$ used in sector $i$ are indicated by $x_{i k}$ and $q_{i k}$, respectively, and the mass of different intermediate goods varieties in each sector is normalized to unity. Figure 1 summarizes the static production structure of the model. Producers in the perfectly competitive service sectors take factor remunerations $w_{i}$

Figure 1: Production structure

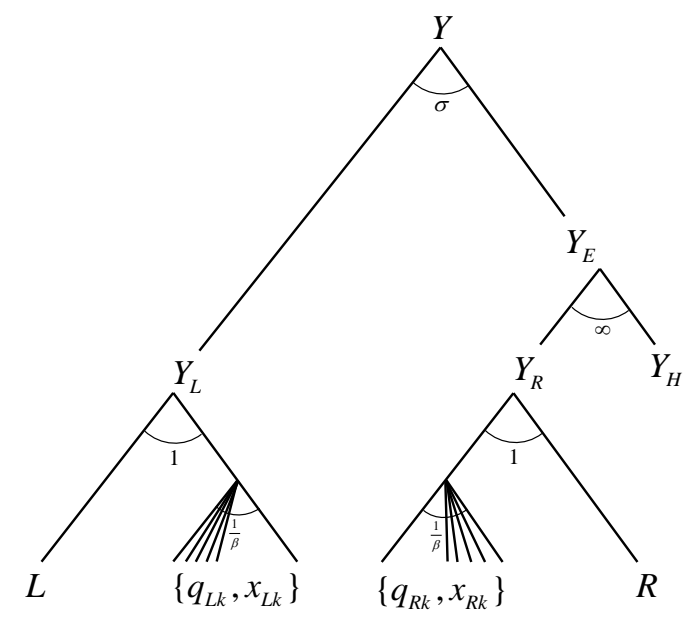

Notes: The branches of the tree denote input flows (flowing from the bottom to the top), whereas the symbols in between the branches denote the different elasticities of factor substitution.

and intermediate goods prices $p_{x i k}$ as given. Their resulting demand for primary inputs and intermediate goods follows from

$$
\begin{aligned}
p_{Y i} \frac{\partial Y_{i}}{\partial M_{i}} & =w_{i}, \\
p_{Y i} \frac{\partial Y_{i}}{\partial x_{i k}} & =p_{x i k} .
\end{aligned}
$$

In the monopolistically competitive intermediate goods sector each firm produces a unique variety and faces a demand function from the service sector, according to (5b). Per unit production costs are equal to $q_{i k}$ units of the final good, so that production costs increase proportionally with quality. Firms invest in R\&D to increase the quality of their products, according to the simplest endogenous growth formulation: ${ }^{8}$

$$
\dot{q}_{i k}=\xi_{i} Q_{i} D_{i k}
$$

where $\xi_{i}$ is a productivity parameter, $Q_{i} \equiv \int_{0}^{1} q_{i k} d k$ is the aggregate quality level in sector $i$, and $D_{i k}$ is labor allocated to R\&D by firm $k$ in sector $i$ at unit cost $w_{D}$. Throughout the

\footnotetext{
${ }^{8}$ Dots above a variable denote time derivatives, i.e., $\dot{x}=d x / d t$, and hats denote growth rates, i.e., $\hat{x}=\frac{d x / d t}{x}$.
} 
rest of the paper, we use the normalization $\xi_{L}^{-1}+\xi_{R}^{-1}=1$, implying that the parameters $\xi_{i}$ reflect relative productivity of labor in both sectors. The level of aggregate research is governed by labor supply $L^{S}$. By choosing specification (6), we follow the literature on energy transitions and directed technical change (cf. Smulders and de Nooij, 2003; Acemoglu et al., 2012; André and Smulders, 2014) and impose a 'standing on shoulders' effect that also drives growth in the 'first-generation' endogenous growth models à la Romer (1990) and Grossman and Helpman (1991): knowledge accumulated in the past makes current researchers more productive, where the aggregate quality level in a sector reflects the stock of sector-specific knowledge. This intertemporal knowledge spillover effect is important for long-run growth possibilities: although production costs rise over time due to quality increases, the cost of innovation declines as well. Our specification (6) implies that both forces exactly offset each other in the long run, implying that the return to innovation and growth can be sustained. ${ }^{9} 10$

The producer of each variety chooses how much to produce and how much to spend on in-house $R \& D$ in order to maximize the net present value of its profits, which gives rise to the following optimality conditions:

$$
\begin{aligned}
p_{x i k} & =\frac{q_{i k} p_{Y}}{1-\beta}, \\
\lambda_{i k} \xi_{i} Q_{i} & \leqq w_{D} \quad \text { with equality if } D_{i k}>0, \\
\frac{\beta}{1-\beta} x_{i k} p_{Y} & =-\dot{\lambda}_{i k}+r \lambda_{i k},
\end{aligned}
$$

where $r$ denotes the nominal interest rate and the $\lambda_{i k}$ 's are the firm's shadow prices of quality in sector $i{ }^{11}$ Price setting equation (7a) shows that firms charge a mark-up over marginal costs. Condition (7b) requires that, at an interior solution, the marginal contribution to firm value of improving quality is equal to its marginal costs. Equation (7c) describes the evolution of the shadow prices of quality. We combine the supply function (7a) with the demand for

\footnotetext{
${ }^{9}$ Our specification with $Q_{i}$ entering linearly in (6) also implies that we end up with a so-called scale effect: an economy with a bigger population grows faster. As a consequence, positive population growth would lead to an ever increasing rate of output growth. Nevertheless, we can easily introduce population growth and eliminate the resulting scale effect by following Howitt (1999): if we assume that the mass of firms in each sector equals $N$ instead of unity, that $N$ grows at the same rate as the population, and that knowledge spillovers depend on the average level of quality per firm, our results are not affected qualitatively. Intuitively, our results are about the incentives to introduce or not the backstop, which are determined by relative costs, while the scale effect is concerned with average costs.

${ }^{10}$ Another simplification in our specification in (6) is that there are no spillovers across sectors. This modeling follows the benchmark case in the literature and is the simplest way to guarantee a balanced growth path along which $R$ and $L$ can grow at different rates while their cost shares remain constant over time and bounded away from 0 and 1 . The case with intersectoral spillovers is discussed at the end of the paper.

${ }^{11}$ Technically, the $\lambda_{i k}$ 's are the co-state variables of the Hamiltonians associated with the problems of the intermediate goods producers (as shown in Appendix A.1.1). For the interpretation of 'shadow prices', we make use of the property that a co-state variable is equal to the marginal value of the relevant state variable, e.g. $\lambda_{i k}=V_{i}^{\prime}\left(q_{i k}\right)$, where $V_{i k}$ denotes the value of firm $i$ in sector $k$ in equilibrium.
} 
intermediate goods varieties (5b) and the production function (4) to find

$$
x_{i k}=x_{i}=\frac{\theta_{i} Y(1-\beta)^{2}}{Q_{i}},
$$

where $i=\{L, R\}$, and the $\theta_{i}$ 's denote the incomes shares of labor and resource services: $\theta_{i} \equiv$ $p_{Y i} Y_{i} / p_{Y} Y{ }^{12}$ This expression implies that all intermediate goods producers within the same sector produce the same output level $x_{i}$. Combining (7b) with (7c) and (8), we get:

$$
r=\beta(1-\beta) \xi_{i} \theta_{i} \frac{Y p_{Y}}{w_{D}}+\hat{w}_{D}-\hat{Q}_{i}, \quad \text { if } D_{i k}>0 .
$$

Equation (9) can be interpreted as a no-arbitrage condition that requires firms to earn the market interest rate on investment in quality improvements. This return depends positively on the relevant income shares $\theta_{i}$ (price effect: quality improvements of relatively scarce factors are more valuable) and on the rate of change in the cost of quality improvements $\hat{w}_{D}-\hat{Q}_{i}$ (capital gain effect: increasing research costs make current improvements more valuable in the future).

By combining (1) with (2a)-(2b), (4), (5b), and (7a), when only the resource is used (i.e., $Y_{H}=0$ ), we can write the production function in terms of quality-augmented primary inputs

$$
Y=(1-\beta)^{2 \frac{1-\beta}{\beta}}\left[\gamma^{\frac{\sigma}{\nu}}\left(L Q_{L}\right)^{\frac{\nu-1}{\nu}}+(1-\gamma)^{\frac{\sigma}{\nu}}\left(R Q_{R}\right)^{\frac{\nu-1}{\nu}}\right]^{\frac{\nu}{\nu-1}}
$$

where $\nu \equiv 1-\beta(1-\sigma)$ so that $\nu \in(0,1)$ because $\sigma \in(0,1)$. Hence, increasing quality in the labor and resource service sectors can be interpreted as labor- and resource-augmenting technical change.

The transversality conditions associated with the problem of firms in the intermediate goods sector are: ${ }^{13}$

$$
\begin{aligned}
& \lim _{t \rightarrow \infty} \lambda_{L}(t) Q_{L}(t) e^{-\int_{0}^{t} r(s) d s}=0, \\
& \lim _{t \rightarrow \infty} \lambda_{R}(t) Q_{R}(t) e^{-\int_{0}^{t} r(s) d s}=0 .
\end{aligned}
$$

The transversality conditions (11a)-(11b) require that the present value of quality in both service sectors vanishes if time goes to infinity. ${ }^{14}$

\footnotetext{
${ }^{12}$ We know from (2a)-(2b) that the factor income shares equal their respective factor output elasticities: $\theta_{i}=$ $\left(\partial Y / \partial Y_{i}\right) \cdot Y_{i} / Y$

${ }^{13}$ See Grass et al. (2008, p. 161) for details concerning the derivation of the transversality conditions.

${ }^{14}$ It follows from (7c)-(8) and the transversality conditions that firms in the same sector face the same shadow prices of quality. Hence, the subscript $k$ is left out and we use aggregate quality levels $Q_{i}$ in (11a)-(11b).
} 


\subsection{Households}

The representative household lives forever, derives utility from consumption of the final good, and inelastically supplies $L^{S}$ units of labor at each moment. It owns the resource stock and all equity in intermediate goods firms. The resource can be extracted from the initial resource stock $S_{0}$, without extraction costs: ${ }^{15}$

$$
\dot{S}=-R, \quad R \geq 0, \quad \int_{0}^{\infty} R(t) d t \leq S_{0}
$$

The household chooses the intertemporal consumption profile to maximize lifetime utility $U(t)=$ $\int_{t}^{\infty} \ln C(z) e^{-\rho(z-t)} d z$, subject to its flow budget constraint $\dot{V}=r V+w_{R} R+w_{L} L^{S}-p_{Y} C$, the resource constraint (12), and the transversality conditions

$$
\begin{gathered}
\lim _{t \rightarrow \infty} \phi_{V}(t) V(t) e^{-\rho t}=0, \\
\lim _{t \rightarrow \infty} \phi_{S}(t) S(t) e^{-\rho t}=0,
\end{gathered}
$$

where $\rho$ denotes the pure rate of time preference, $V$ is (non-resource) assets holdings, and $\phi_{V}$ and $\phi_{S}$ are the shadow prices of asset holdings and resource wealth, respectively. In the resource constraint, we have used that labor is perfectly mobile between the production and the research sector, which implies a uniform wage rate in equilibrium: $w_{L}=w_{D}$.

Optimizing behavior of the household gives rise to the following two familiar conditions:

$$
\begin{aligned}
\hat{C} & =r-\hat{p}_{Y}-\rho, \\
\hat{w}_{R} & =r .
\end{aligned}
$$

Condition (14a) is the Ramsey rule, which relates the growth rate of consumption to the difference between the real interest rate and the pure rate of time preference. Condition (14b) is the Hotelling rule, which requires the resource price to grow at the interest rate so that resource owners are indifferent between extracting and conserving an additional unit of the resource.

\subsection{Goods and factor market equilibrium}

The goods market equilibrium condition is given by:

$$
Y=C+\int_{0}^{1} q_{L k} x_{L k} d k+\int_{0}^{1} q_{R k} x_{R k} d k+Y_{H} / \eta
$$

\footnotetext{
${ }^{15}$ We abstract from extraction costs. Introduction of constant per unit extraction costs (lower than the per unit price of the backstop) will not change our results qualitatively. Introduction of stock-dependent extraction costs can lead to an equilibrium with incomplete exhaustion of the resource stock (cf. Van der Ploeg and Withagen, 2014).
} 
From (8) we write the goods market equilibrium as

$$
C=\left[1-\left(1-\theta_{E}+\theta_{R}\right)(1-\beta)^{2}-\left(\theta_{E}-\theta_{R}\right)\right] Y,
$$

where $\theta_{E} \equiv p_{Y E} Y_{E} / p_{Y} Y$ is the energy income share and $\theta_{E}-\theta_{R}$ is the income share of the backstop. ${ }^{16}$

Labor market equilibrium requires that labor supply $L^{S}$ equals labor demand from the labor service sector and from R\&D:

$$
L^{S}=L+D_{L}+D_{R}
$$

where $D_{i} \equiv \int_{0}^{1} D_{i k} d k$ is aggregate research effort in sector $i$. We use the labor market equilibrium condition together with (5a) to obtain

$$
L^{S}-D_{L}-D_{R}=\beta\left(1-\theta_{E}\right) \frac{L^{S}}{\mu_{L}}
$$

where $\mu_{L} \equiv w_{L} L^{S} / p_{Y} Y$ denotes the 'full labor income share', including labor income from research.

\section{Dynamics of the model}

The model features three state variables: the two knowledge stocks $Q_{L}$ and $Q_{R}$, and the resource stock $S$. To facilitate the analysis of the dynamics, we condense the model to a fourdimensional block-recursive dynamical system in the energy income share $\theta_{E}$ (which equals $\theta_{R}$ when fossil is used), the full labor income share $\mu_{L}$, the effective stock rate $Z \equiv Q_{R} S /\left(Q_{L} L^{S}\right)$, and the marginal benefit-cost rate of research in the resource service sector $\chi \equiv \xi_{R} Q_{R} \lambda_{R} / w_{L}$. The system is block-recursive in the sense that the $\left(\theta_{R}, \mu_{L}, \chi\right)$-subsystem can be solved (almost) independently from the $Z$-subsystem. Only in the last stage of the solution procedure, the differential equation for $Z$ needs to be solved in order to identify the initial points. The advantage of the decomposition into subsystems is that a large part of the transitional dynamics can be illustrated by phase diagrams in the $\left(\theta_{R}, \mu_{L}\right)$-plane. These two variables have a clear economic interpretation, and other macroeconomic variables of interest can be expressed in terms of $\theta_{R}$ and $\mu_{L}$, as shown in Appendix A.1.6.

Because the resource and the backstop are perfect substitutes, the economy uses only the cheapest of the two. Below, we first separately characterize the equilibrium with fossil use and

\footnotetext{
${ }^{16}$ Note that $\theta_{L}+\theta_{E}=1$ because of constant returns; without backstop we have $\theta_{L}+\theta_{R}=1$; without fossil use, we have $\theta_{R}=0$.
} 
the one with backstop use, before we discuss the potential transition between the two and the role of expectations.

\subsection{The fossil economy}

In the fossil economy, the backstop technology will not be used, implying that $\theta_{E}=\theta_{R}>0$. Using (16) we derive that consumption is a fixed fraction of output: $C=\left[1-(1-\beta)^{2}\right] Y$. Imposing $Y_{H}=0$ in the relative factor demand from the final good sector obtained from (2a)(2b) and using (4), (5a), and (8), we find

$$
\frac{\theta_{R}}{1-\theta_{R}}=\left(\frac{w_{R}}{w_{L}} \frac{Q_{L}}{Q_{R}}\right)^{1-\nu}\left(\frac{1-\gamma}{\gamma}\right)^{\sigma}
$$

Converting (19) into growth rates and using the Hotelling rule (14b), we obtain:

$$
\hat{\theta}_{R}=(1-\nu)\left(1-\theta_{R}\right)\left[r-\hat{w}_{L}+\hat{Q}_{L}-\hat{Q}_{R}\right] .
$$

Equation (20) implies that the resource income share increases if, after correcting for relative productivity changes, the resource price grows faster than the wage rate.

We find the following useful expression for resource extraction growth from converting (5a) into growth rates and substituting (14a), (14b), and $\hat{C}=\hat{Y}$ :

$$
\hat{R}=\hat{\theta}_{R}-\rho,
$$

which implies that resource extraction grows if the resource income share increases fast enough.

The fossil economy may be characterized by different technological regimes: one in which there is both labor- and resource-augmenting technical change and another without resourceaugmenting technical change, which we will now discuss separately. ${ }^{17}$

\subsubsection{Dynamics with both types of technical change}

The fossil economy with both types of research occurring in equilibrium evolves as follows:

Lemma 1 The dynamics of the fossil economy with labor-and resource-augmenting technical change are described by the following two-dimensional system of first-order differential equations

\footnotetext{
${ }^{17}$ We leave out a discussion of the corner solution without labor-augmenting technical change, because it is not essential for understanding the energy transition. It does not arise if the first condition in footnote 18 holds along all equilibrium paths that we derive. This requires only a mild condition on parameters, such that the economy has sufficient growth potential $\left(\xi_{L} L^{S}\right.$ large relative to $\rho$ ).
} 
in the full labor income share $\mu_{L}$ and the resource income share $\theta_{R}$ :

$$
\begin{aligned}
& \dot{\mu}_{L}=\left(L^{S}+\rho\right) \mu_{L}-L^{S} \beta\left(2-\beta-\theta_{R}\right), \\
& \dot{\theta}_{R}=\frac{\beta L^{S} \theta_{R}\left(1-\theta_{R}\right)(1-\nu)}{\mu_{L}}\left\{1+(1-\beta)\left(1+\xi_{L}\right)-\frac{\mu_{L}}{\beta}-\left[1+(1-\beta) \xi_{L} \xi_{R}\right] \theta_{R}\right\} .
\end{aligned}
$$

Proof. See Appendix A.1.3.

Panel (a) of Figure 2 shows the phase diagram in the $\left(\theta_{E}, \mu_{L}\right)$-plane for the dynamical system of the fossil economy with both types of research. The linear $\dot{\mu}_{L}=0$ and $\dot{\theta}_{R}=0$ loci are derived from (22a)-(22b). The intersection of these two loci determines the unique steady state of the fossil economy with both types of technical progress. There is a unique trajectory leading to the steady state at point $\mathrm{D}^{\prime \prime}$, indicated by the fat red dotted line in the figure. ${ }^{18}$ Along the saddle path, two counteracting forces affect the resource income share. On the one hand, increasing physical scarcity of the resource puts upward pressure on the resource income share. On the other hand, induced resource-augmenting technical change puts downward pressure on the resource income share. These opposing effects exactly offset each other in the steady-state equilibrium, resulting in the constant long-run income shares ${ }^{19} 20$

$$
\begin{aligned}
\theta_{R}^{*} & \equiv \xi_{R}^{-1}+\frac{\left(1-\beta+\xi_{L}^{-1}\right) \rho}{\xi_{L} \xi_{R}(1-\beta)\left(L^{S}+\rho\right)+\rho} \\
\mu_{L}^{*} & \equiv \frac{\beta L^{S}}{L^{S}+\rho}\left(2-\beta-\xi_{R}^{-1}-\frac{\left(1-\beta+\xi_{L}^{-1}\right) \rho}{\xi_{L} \xi_{R}(1-\beta)\left(L^{S}+\rho\right)+\rho}\right) .
\end{aligned}
$$

To illustrate that resource use may peak, panel (a) of Figure 2 also shows the $\hat{R}=0$ isocline (derived from (21)), which is located below the $\dot{\theta}_{R}=0$ locus. ${ }^{21}$ At points below (above) the $\hat{R}=0$ isocline, resource extraction is growing (declining) over time. Hence, an economy moving along the saddle path necessarily exhibits decreasing resource use in the long run.

\footnotetext{
${ }^{18}$ This trajectory is only applicable for combinations of $\theta_{R}$ and $\mu_{L}$ for which both types of research are nonnegative. By combining (6), (9), and (18), we find that $D_{L} \geq 0$ and $D_{R} \geq 0$ require $\mu_{L} \geq \beta\left[2-\beta-(1-\beta) \xi_{L}\right]-$ $\beta\left[1-(1-\beta) \xi_{L}\right] \theta_{R}$ and $\mu_{L} \geq \beta(2-\beta)-\beta\left[1+(1-\beta) \xi_{R}\right] \theta_{R}$, respectively.

${ }^{19}$ In the long run, the income share of resources is constant over time, but at a level that is endogenous. For example, a higher discount rate $\rho$ ceteris paribus increases both the depletion rate $R / S$ and the resource share $\theta_{R}$ in the long run. Thus our model is not subject to Hart's (2013) critique that income shares are independent of resource scarcity in a class of models of directed technical change with 'extreme state dependence' (i.e., the productivity of each sector's innovation is merely affected by the aggregate quality level in their sector, and not by the aggregate quality level in the other sector).

${ }^{20}$ This fossil steady state is feasible if the associated research levels in both the labor and resource service sector are non-negative. Substituting (23a)-(23b) into the expressions in footnote 18 we find that whereas for the resource service sector the steady-state condition is always met, for the labor service sector $D_{L}^{*} \geq 0$ requires $\xi_{L} L^{S}>\rho\left(\xi_{L}+\beta /(1-\beta)\right)$.

${ }^{21}$ The expression for the $\hat{R}=0$ isocline is derived in Appendix A.1.7.
} 
Figure 2: Phase diagrams - Fossil economy

(a) Both types of technical change

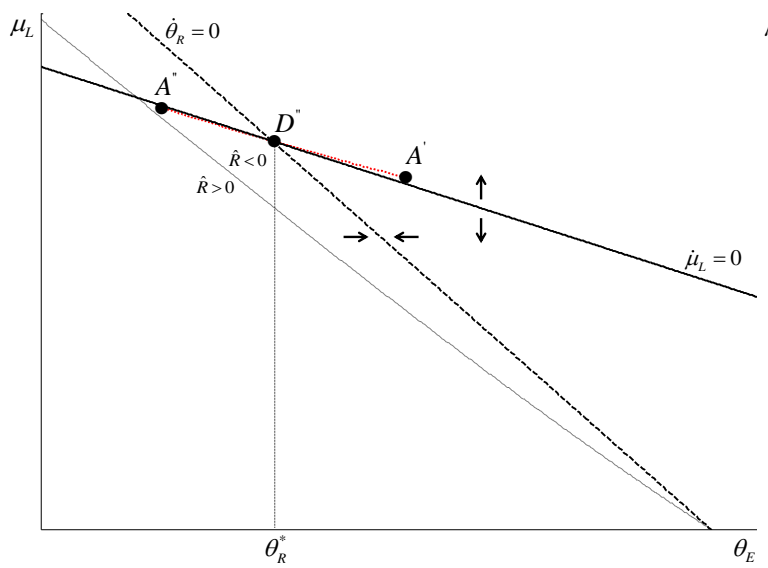

(b) Purely labor-augmenting technical change

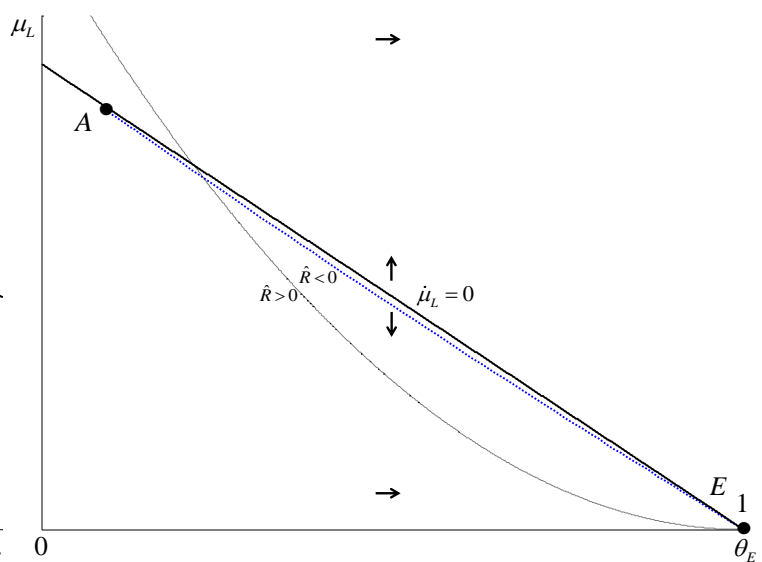

Notes: The solid and dashed black lines represent the $\dot{\mu}_{L}=0$ and $\dot{\theta}_{R}=0$ loci, respectively. The dotted black line is the extraction isocline. The red and blue fat-dotted lines represent the saddle paths that lead to the steady state at point $\mathrm{D}^{\prime \prime}$ in panel (a) and at point $\mathrm{E}$ in panel (b), respectively. The steady state value of $\theta_{R}$ in panel (a) is denoted by $\theta_{R}^{*}$.

\subsubsection{Dynamics with purely labor-augmenting technical change}

The fossil economy with purely labor-augmenting technical change (i.e., $D_{L}>0$ and $D_{R}=0$ ) evolves as follows:

Lemma 2 The dynamics of the fossil economy with purely labor-augmenting technical change are described by the following two-dimensional system of first-order differential equations in the full labor income share $\mu_{L}$ and the resource income share $\theta_{R}$ :

$$
\begin{aligned}
& \dot{\mu}_{L}=\left(\xi_{L} L^{S}+\rho\right) \mu_{L}-\xi_{L} L^{S} \beta(2-\beta)\left(1-\theta_{R}\right), \\
& \dot{\theta}_{R}=(1-\nu) \xi_{L} \frac{L^{S} \beta(1-\beta)}{\mu_{L}} \theta_{R}\left(1-\theta_{R}\right)^{2} .
\end{aligned}
$$

Proof. See Appendix A.1.4.

Panel (b) of Figure 2 shows the phase diagram in the $\left(\theta_{E}, \mu_{L}\right)$-plane for the dynamical system of the fossil economy with purely labor-augmenting technical change. The linear $\dot{\mu}_{L}=0$ locus is derived from (24a). The $\dot{\theta}_{R}=0$ locus is left out, because $\dot{\theta}_{R}>0$ for all relevant values of $\theta_{R} \in(0,1)$. The figure shows that, in case the backstop technology is never introduced and resource-augmenting technical change never takes place, the unique steady state is located at the intersection of the $\dot{\mu}_{L}=0$ locus and the vertical $\theta_{E}=1$ line denoted by point $\mathrm{E}$ in the figure. There is a unique path leading to point $\mathrm{E}$, see the fat dotted blue line in the figure. Along the equilibrium path to point $\mathrm{E}$ the energy income share is increasing over time, because of increasing physical scarcity of the resource. Without resource-augmenting technical change, 
there is no offsetting force, so that the energy income share approaches unity in the long run. The figure also shows the $\hat{R}=0$ isocline (derived in Appendix A.1.7), which hits the steady state at point E. At points below (above) the $\hat{R}=0$ isocline, resource extraction is growing (declining) over time. Hence, in the long run, i.e., close to the steady state point E, resource extraction is declining, but resource use might initially increase. ${ }^{22}$

\subsection{The backstop economy}

Once the economy has permanently switched from fossil fuels to the backstop technology, we have $Y_{R}=D_{R}=0$ and $\theta_{E}=p_{Y H} Y_{H} / p_{Y} Y$. Lemma 3 states that the energy income share and the full labor income share are constant in this backstop regime.

Lemma 3 In the backstop regime, the energy income share $\theta_{E}$ and the full labor income share $\mu_{L}$ are constant over time and equal to, respectively

$$
\begin{aligned}
& \theta_{E}=(1-\gamma)^{\sigma} \eta^{\sigma-1} \equiv \theta_{H}^{*}, \\
& \mu_{L}=\frac{\xi_{L} L^{S}}{\xi_{L} L^{S}+\rho} \beta(2-\beta)\left[1-(1-\gamma)^{\sigma} \eta^{\sigma-1}\right] \equiv \mu_{L}^{+} .
\end{aligned}
$$

Proof. See Appendix A.1.5.

Energy generation with the backstop technology uses the final good $Y$ as input. As a result, labor is the only primary factor of production, implying that technical change is effectively neutral in the backstop economy. It follows that the economy immediately settles down at its steady state equilibrium with constant income shares and a constant amount of research.

\subsection{The energy transition}

Since energy services from fossil and those from the backstop technology are perfect substitutes, only the cheapest of the two will be deployed at a particular moment in time. ${ }^{23}$ Resources are a cheaper energy source than the backstop if and only if $\theta_{E}<\theta_{H}^{*}$, as can be derived from (2a)(2b). Intuitively, when energy users spend a small share of total cost on energy, the marginal product of energy is too small to warrant spending on the relatively expensive backstop.

We now turn to the question whether fossil is going to be replaced by the backstop in equilibrium and the question which of the three steady states that are associated with the three regimes discussed above can arise in equilibrium. To make the backstop relevant for our analysis, we naturally need to assume that the backstop can be profitable in the long run,

\footnotetext{
${ }^{22}$ The slope of the $\hat{R}=0$ isocline is zero at the steady state so that the saddlepath cuts the isocline from above.

${ }^{23}$ Appendix A.1.8 shows that simultaneous use will not take place in equilibrium.
} 
which requires $(1-\gamma)^{\sigma} \eta^{\sigma-1} \equiv \theta_{H}^{*}<1$. A steady-state equilibrium with fossil and purely laboraugmenting technical change, as represented by point E in Figure 2(b), can then no longer arise, since backstop energy becomes cheaper than fossil before this steady state is reached. However, the fossil steady state with both types of innovation, as represented by point D" in Figure 2(a), can potentially be an equilibrium, if the following necessary condition is satisfied:

Lemma 4 A necessary condition for the existence of multiple equilibria is $\theta_{R}^{*} \leq \theta_{H}^{*}<1$.

Proof. If $\theta_{H}^{*}<\theta_{R}^{*}$, the path leading to the steady state of the fossil economy $\left(\theta_{R}^{*}, \mu_{L}^{*}\right)$ along which fossil fuels are used forever cannot be a competitive equilibrium, because in the interval with $\theta_{R}>\theta_{H}^{*}$, the backstop technology is relatively cheaper than fossil fuels so that a shift to the backstop technology must occur.

In principle the price of fossil services changes over time, with a tendency to increase because of a growing scarcity rent and a tendency to decrease because of resource-augmenting technical change; hence, if on balance the price keeps increasing, the resource income share increases and a transition from fossil fuel to the backstop technology might occur. Since the model is forward looking, we need to start in the steady state and see how we can reach this state, by first investigating the possible sequence of regime switches.

The first regime switch we characterize is the one to the backstop regime. Since the backstop economy is always in the steady state, the economy permanently stops using fossil once it enters the backstop regime. The direct implication is that not only in the backstop economy but also in the periods immediately before the transition towards it, resource-augmenting innovation is not profitable: the period of resource use is too short to recoup the investment costs. At the moment of the energy switch, energy generation with the backstop technology jumps up from zero to a positive level, which comes at the cost of consumption unless output discretely jumps up. However, consumption cannot jump in equilibrium since consumers are forward looking and smooth consumption. ${ }^{24}$ Using the goods market equilibrium condition (16) and denoting the switching time by $T$, we find that $Y\left(T^{-}\right)=\left(1-\theta_{H}^{*}\right) Y\left(T^{+}\right)$is required to prevent a discontinuity in consumption. ${ }^{25}$ From this condition, we get that the full labor income share jumps down according to (18). The following lemma formalizes these results.

Lemma 5 (i) The economy can only enter the backstop regime from an equilibrium with $D_{R}=0$. (ii) At $T$, the moment of the switch from fossil fuels to the backstop technology, the full labor income share jumps down from $\mu_{L}^{-}=\frac{\xi_{L} L^{S}}{\xi_{L} L^{S}+\rho} \beta(2-\beta)$ to $\mu_{L}^{+}=\left(1-\theta_{H}^{*}\right) \mu_{L}^{-}$.

\footnotetext{
${ }^{24}$ The Ramsey rule (14a) implies that consumption is continuous as long as the rate of interest is finite, which is the case according to (9) with $i=L$.

${ }^{25}$ We use $x^{-}\left(x^{+}\right)$as short-hand notation for $x\left(T^{-}\right)\left(x\left(T^{+}\right)\right)$, where $x\left(T^{-}\right) \equiv \lim t \uparrow T(t)$ and $x\left(T^{+}\right) \equiv$ $\lim _{t \downarrow T} x(t)$.
} 
Proof. See Appendix A.1.9.

The other two regime switches we characterize are the ones between simultaneous R\&D and purely labor-augmenting R\&D. From the same logic as stated above, an equilibrium with simultaneous R\&D cannot last forever if investors expect fossil use to stop after a certain moment: resource-augmenting innovations stay profitable only if the period over which they are going to be applied is long enough. In contrast, entering the simultaneous $R \& D$ regime from the purely labor-augmenting R\&D regime, the last regime switch we need to characterize,${ }^{26}$ must happen as soon as the return to resource-augmenting innovation starts to equal the return to labor-augmenting innovation. The following Lemma characterizes these two remaining possible regime switches, by using our definition of the benefit-cost ratio of research in the resource service sector $\chi \equiv \xi_{R} Q_{R} \lambda_{R} / w_{L}$ :

Lemma 6 At the instant the economy enters or leaves the simultaneous-R\&D regime, $\chi=1$. Furthermore, at the instant the economy enters (leaves) the simultaneous- $R \& D$ regime, $\mu_{L}=(>$ ) $\beta(2-\beta)-\beta\left[1+(1-\beta) \xi_{R}\right] \theta_{R}, D_{R}=(>) 0$, and $\dot{\chi}=(<) 0$.

Proof. See Appendix A.1.10.

We now show how the transitions can be characterized by pointing out how the phase diagrams introduced above are connected in equilibrium. We consider the case in which the initial resource stock is large enough to warrant an initial equilibrium resource income share at the fossil path that is smaller than the steady-state resource income share of the fossil economy, i.e., $\theta_{R}(0)<\theta_{R}^{*}{ }^{27}$ Moreover, we assume that labor supply is large enough to obtain positive labor-augmenting technical change along the entire transition paths.

Panels (a) and (b) of Figure 3 show the phase diagrams of the two technological regimes in the fossil economy. The figures contain lines for the $\dot{\mu}_{L}=0$ and $\dot{\theta}_{R}=0$ loci, which are derived from Lemma 1 and 2, respectively. Panel (a) also shows the non-negativity constraint $D_{R}=0$ below which resource-augmenting technical change equals zero, which follows from the second condition in footnote 18. The fat dots represent the transition paths. The letters $\mathrm{B}$ and $\mathrm{C}$, which occur in both panels (a) and (b) at the borders of the shaded areas, indicate

\footnotetext{
${ }^{26}$ Since the backstop economy is always in the steady state, this regime is absorbing, i.e., there are no regime switches out of the backstop regime. Lemma 5 shows that a regime switch from simultaneous R\&D to the backstop cannot occur. Hence, while we have three regimes, and hence six regime switches in principle, there are only three of them possible in equilibrium. Recall that we ruled out an equilibrium with no labor-augmenting innovation by choosing $\xi_{L} L^{S}$ sufficiently high relative to $\rho$.

${ }^{27}$ The relationship between the initial stock of fossil fuels and the initial resource income share will be discussed in Section 3.4. Intuitively, a higher $S_{0}$ implies less resource scarcity in the beginning, a lower relative resource price, and therefore a lower initial income share (if resource and labor services are gross complements, which we have assumed).
} 
Figure 3: Phase diagrams - Transition to backstop steady state

(a) Both types of technical change

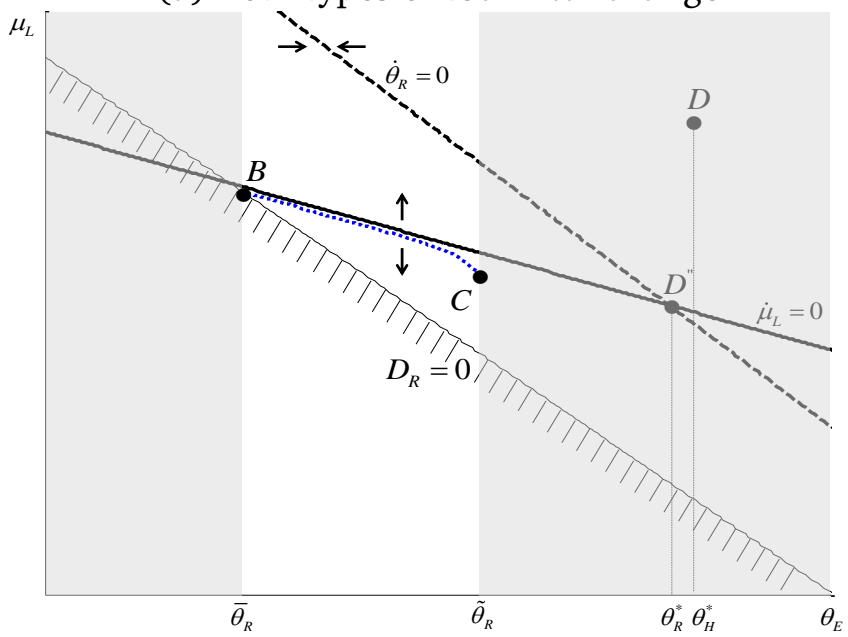

(b) Purely labor-augmenting technical change

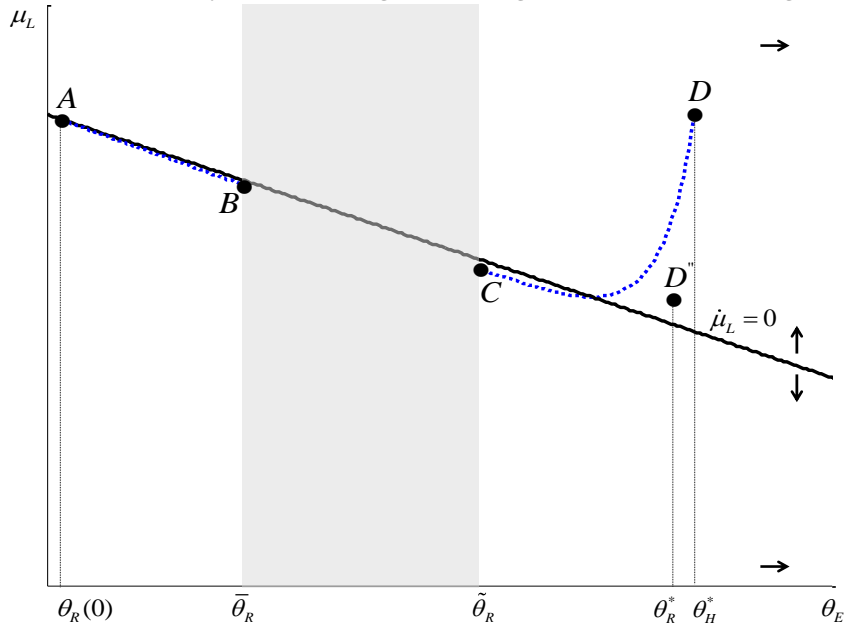

(c) Benefit-cost ratio

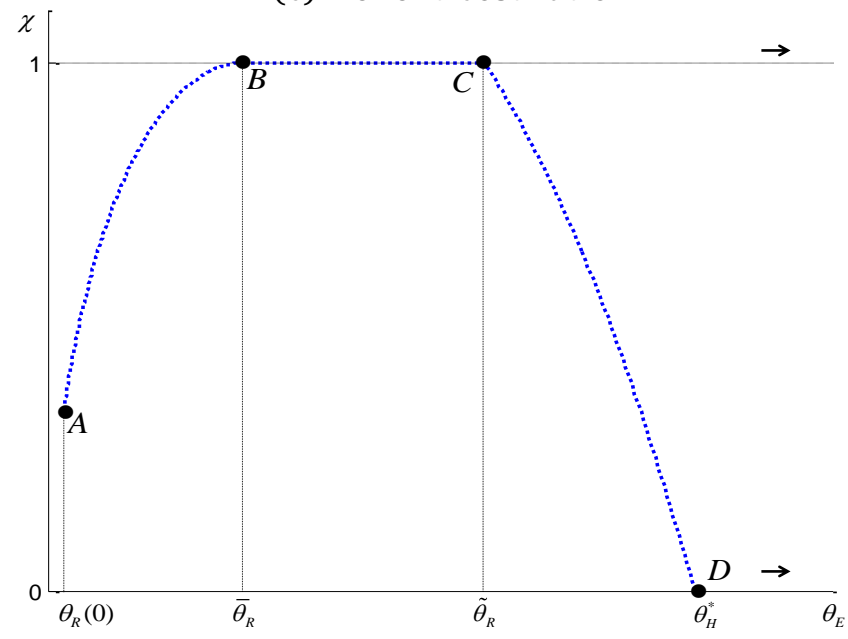

Notes: The fat dots represent the transitional path. Dynamics in the shaded areas are not applicable to the scenario in which the backstop technology will be implemented. As long as $\theta_{E}<\theta_{H}^{*}$, it holds that $\theta_{R}=\theta_{E}$. 
the level of the energy share for which the economy switches from one technological regime to the other. We are going to show that in equilibrium the economy may go through different regimes subsequently. Figure 3 illustrates the case with $\theta_{R}^{*}<\theta_{H}^{*}<1$, the necessary condition for multiple equilibrium paths (cf. Lemma 4).

The backstop steady state occurs at $\theta_{E}=\theta_{H}^{*}$. The transition path leading to this steady state is represented by the blue fat-dotted line. It starts at point A in panel (b), then moves along the blue fat-dotted line shown in the same panel to point $\mathrm{B}$, continues along the blue fat-dotted line in panel (a) from point $\mathrm{B}$ to point $\mathrm{C}$ and finally moves on along the blue fat-dotted line in panel (b) from point $\mathrm{C}$ to point $\mathrm{D}$. In accordance with Lemma 5, the labor share jumps at point D when the economy switches to the backstop to ensure a continuous consumption path. Points $\mathrm{B}$ and $\mathrm{C}$ are determined by using backward induction.

At the moment of the switch to the backstop technology $\chi$ equals zero, because resourceaugmenting technologies are worthless from that moment onwards. Working backwards in time, the differential equation for $\chi$, which is derived in Appendix A.1.10, can be used, together with the time paths for $\theta_{E}$ and $\mu_{L}$ obtained from the solution to the dynamical system in Lemma 2 , to find the transition path in $\left(\theta_{E}, \chi\right)$-space that leads to the point $\left(\theta_{H}^{*}, 0\right)$. This final point is indicated by $\mathrm{D}$ in panel (c) of the figure. We use this transition path to determine the point at which $\chi$ becomes equal to unity, point $\mathrm{C}$, where resource-augmenting technical change becomes profitable, implying that the simultaneous instead of the purely labor-augmenting R\&D regime applies. Still working backwards in time, we use the result in Lemma 6 to find that the starting point of the simultaneous $R \& D$ regime is located in the point where the transition path crosses the $D_{R}=0$ line in panel (a), indicated by point $\mathrm{B}$. This gives the value of $\theta_{E}$ in panel (c) at which $\chi$ reaches the value 1 . To the left of point $\mathrm{B}$, we again make use of the differential equation for $\chi$ to construct the remainder of the transition path in $\left(\theta_{E}, \mu_{L}\right)$-space.

The resulting blue-dotted line in panel (c) shows that the benefit-cost ratio of resourceaugmenting innovation, $\chi$, is smaller than unity in between point $\mathrm{A}$ and point $\mathrm{B}$, equal to unity on the path from point $\mathrm{B}$ to point $\mathrm{C}$, and again smaller than unity along the path from point C to point D. Accordingly, during the transition from fossil fuels to the backstop technology, resource-augmenting technical change is positive only on the transition path between points $B$ and C. With the resource income share $\theta_{R}$ lower than $\bar{\theta}_{R}$, fossil fuel is relatively too cheap to warrant investments in resource-augmenting technical change. With the resource income share $\theta_{R}$ exceeding $\tilde{\theta}_{R}$, the switch from fossil fuel to the backstop technology occurs soon, so that investing in resource-augmenting technical change is not profitable anymore (cf. Lemma 5). This explains why the transition in the $\left(\theta_{E}, \mu_{L}\right)$-plane starts in panel (b), continues in panel (a), and finally switches back to panel (b). 
Figure 4: Phase diagrams - Transition to fossil steady state

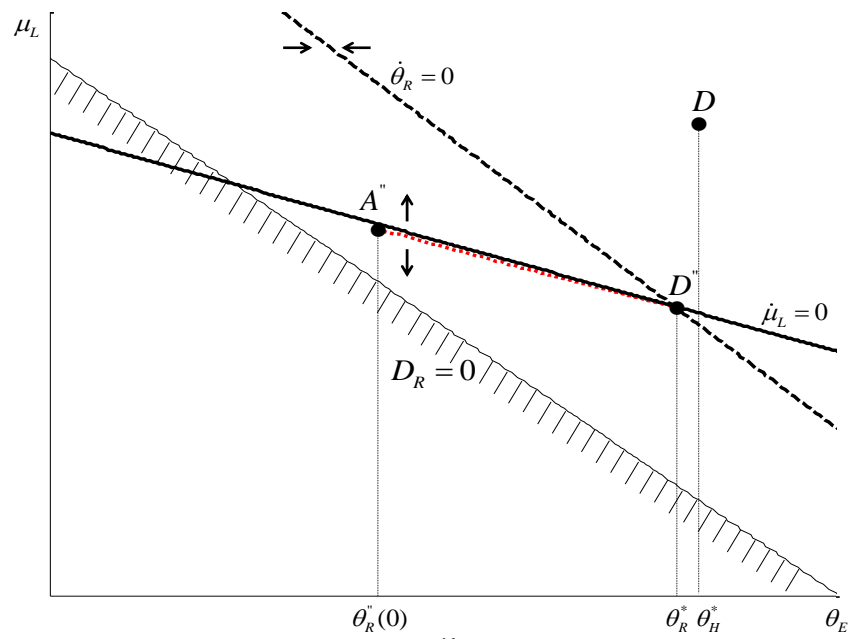

Notes: The fat dots represent the transitional path to the steady state at point $\mathrm{D}^{\prime \prime}$.

Figure 4 shows the transition to the fossil steady state, i.e., point $\mathrm{D}^{\prime \prime}$, which has both types of technical change. Above the $D_{R}=0$ line the transition path that leads to this steady state follows the dynamics of Lemma 1 ; it is represented by the red fat-dotted line $\mathrm{A}^{\prime \prime} \mathrm{D}^{\prime \prime}$. For low $\theta_{E}$, i.e., below the $D_{R}=0$ line, the economy must be in the purely labor-augmenting regime (cf. Lemma 6). Along the transition path, resource-augmenting technical change still occurs in the long run and energy generation will rely upon fossil fuels forever: since the steady-state resource income share, $\theta_{R}^{*}$, is smaller than the equilibrium backstop share, $\theta_{H}^{*}$, the resource remains cheaper than the backstop technology in the steady state.

Summarizing, we have identified two transition paths in Figures 3 and 4. Along the first one, resource-augmenting technical change will drop to zero eventually, implying that the resource income share continues growing beyond $\theta_{R}^{*}$ until it reaches $\theta_{H}^{*}$ at the moment the backstop technology is introduced. The second transition path is characterized by both types of technical change in the long run, so that the backstop technology will not become competitive as the resource income share converges to $\theta_{R}^{*}$ which is smaller than $\theta_{H}^{*}$.

\subsection{Equilibrium paths and self-fullfilling prophecies}

What remains to be determined is where the economy would start on the paths characterized in Section 3.3 (fossil and backstop), i.e., what would be the initial prices given the initial state variables. Clearly the fossil path must start with initial prices such that the resource stock is never exhausted, while initial prices at the backstop path must ensure full extraction before the time at which the backstop regime is entered. We will characterize what the fossil path and the backstop path imply for depletion of the resource stock and growth of the knowledge stocks and 
then infer from the initial values of these stocks where the equilibrium path must start. This analysis will confirm the possibility of two equilibrium paths, which we will state in advance as follows:

Proposition 1 (i) if $\theta_{H}^{*}<\theta_{R}^{*}$, the unique equilibrium in a decentralized economy is a transition from fossil fuels to the backstop technology; (ii) if $\theta_{H}^{*} \geq \theta_{R}^{*}$, then for a non-empty range of parameters and initial conditions, the market equilibrium has two steady states and self-fulfilling expectations can lead the economy either to the fossil or to the backstop steady state.

Proof. Part (i) follows immediately from Lemma 1 and Lemma 4. Since part (ii) only gives a necessary condition, we still need to show that the multiplicity arises in an example; this is done in the analysis below.

To connect the dynamics of the resource share derived above to the dynamics of the state variables we define the initial effective stock rate $Z \equiv S Q_{R} / L^{S} Q_{L}$ and construct a phase diagram in the $\left(Z, \theta_{E}\right)$-plane. Total differentiation of $Z$ gives

$$
\dot{Z}=Z\left(\hat{Q}_{R}-\hat{Q}_{L}\right)-\left(\frac{1-\gamma}{\gamma}\right)^{\frac{\sigma}{1-\nu}}\left(\frac{1-\theta_{E}}{\theta_{E}^{\nu}}\right)^{\frac{1}{1-\nu}} \frac{\beta}{\mu_{L}},
$$

where we have used (4) and (19) to express $\hat{S}=-R / S$ in terms of $\theta_{E}$ and $\mu_{L}$. We define $\mu_{L}=f\left(\theta_{E}\right)$ as the fossil equilibrium path in $\left(\theta_{E}, \mu_{L}\right)$-space derived in Section 3.3 and use it in (22b), (24b), and (26), to construct the saddle path leading to the fossil steady state at point $\mathrm{D}^{\prime \prime}$ in $\left(Z, \theta_{E}\right)$-space, as shown by the red fat-dotted line in the phase diagram in panel (a) of Figure 5. The figure also shows the isoclines $\dot{Z}=0$ (solid line) and $\dot{\theta}_{R}=0$ (dashed line) for the fossil regime with both types of technical change, which are derived from (22b) and (26) with $\mu_{L}=f\left(\theta_{E}\right)$ imposed. The backstop path, which is shown in the figure by the blue fat-dotted line leading to point $\mathrm{D}$, can be constructed in a similar way. ${ }^{28}$ The initial income shares at the two equilibrium paths, $\theta_{R}^{\prime \prime}(0)$ and $\theta_{R}(0)$, can be determined in panel (a) of Figure 5 by finding the intersection point of the two equilibrium paths with the vertical $Z=Z(0)$-line, i.e., point $\mathrm{A}^{\prime \prime}$ and point $\mathrm{A}$, respectively.

While our previous figures separate the transition path to the backstop steady state from the transition path to the fossil steady state, Figure 5 shows both paths in the same plane and allows to determine the starting point of each of the two paths. The phase diagrams are the counterparts of the central figures in Krugman (1991) and Matsuyama (1991), who also construct

\footnotetext{
${ }^{28}$ We construct the backstop path in $\left(Z, \theta_{E}\right)$-space by defining $\mu_{L}=g\left(\theta_{E}\right)$ as the backstop path in $\left(\theta_{E}, \mu_{L}\right)$-space derived in Section 3.3 and by using $\mu_{L}=g\left(\theta_{E}\right)$ in (22b), (24b), and (26). Note that the $\dot{Z}=0$ locus and the $\dot{\theta}_{R}=0$ locus are not valid for the transition to the backstop steady state, because we have used $\mu_{L}=f\left(\theta_{E}\right)$ to construct them.
} 
Figure 5: Phase diagrams in $\left(Z, \theta_{E}\right)$-space

(a) Cheap backstop (high $\eta$ )

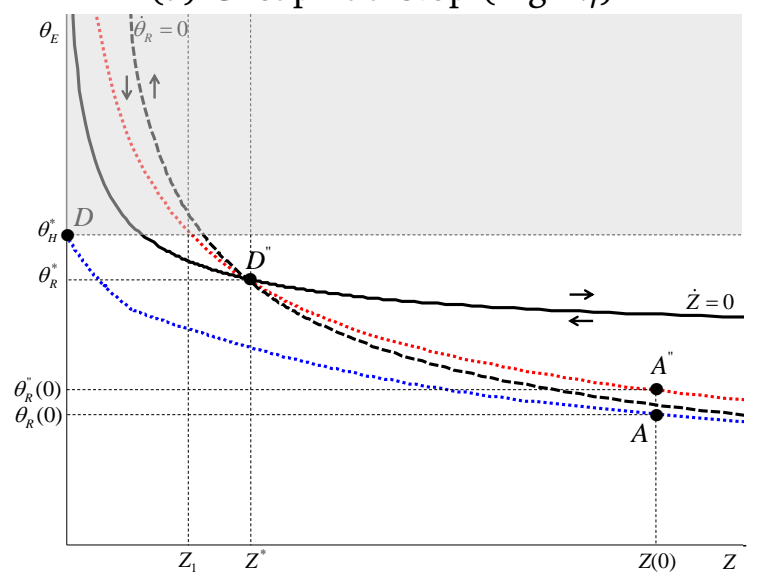

(b) Expensive backstop (low $\eta$ )

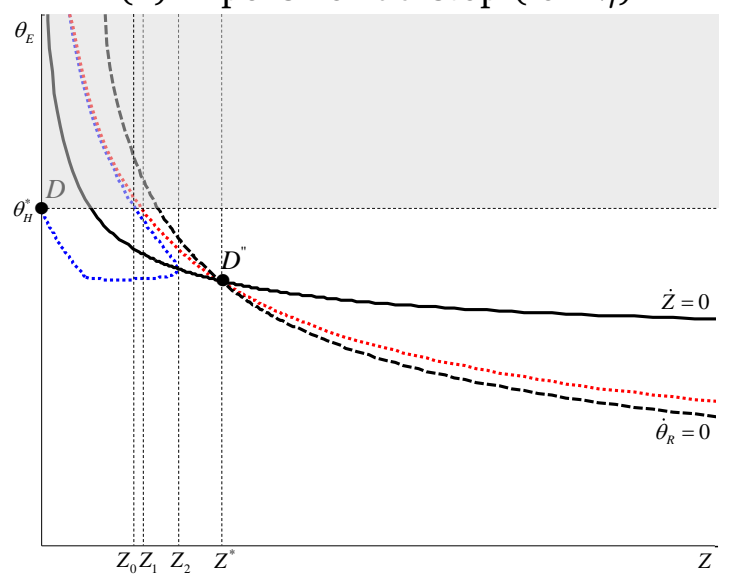

Notes: The isoclines $\dot{Z}=0$ (solid line) and $\dot{\theta}_{R}=0$ (dashed line) are derived from (26) and (22b) with $\mu_{L}=f\left(\theta_{E}\right)$ imposed. The red fat-dotted line represents the transitional path to the fossil steady state at point $\mathrm{D}^{\prime \prime}$. The blue fat-dotted line represents the transitional path to the backstop steady state at point D.

phase diagrams with a predetermined variable on the horizontal and a non-predetermined variable on the vertical axis. ${ }^{29}$ Along the equilibrium path starting at $\mathrm{A}^{\prime \prime}$ and leading to the fossil steady state $\mathrm{D}^{\prime \prime}$ in panel (a) of the figure, investors rationally expect that the backstop technology will never be implemented, because the resource will always remain cheaper. As a result, investment in resource-augmenting technologies will remain attractive, implying that rapid resource-augmenting technical change indeed ensures that the resource will not be driven out of the market by the backstop technology: expectations turn out to be truly rational along the saddle path towards the fossil steady state. Similarly, along the equilibrium path starting at point $\mathrm{A}$ and leading to the backstop steady state at point $\mathrm{D}$, investors rationally expect the backstop technology to become competitive in the future, because resource-augmenting technical change will not be strong enough to prevent the backstop technology from entering the market, due to a growing resource price over time. As a result, investment in resource-saving technology is less attractive and even drops to zero at a certain moment, when the expected implementation of the backstop technology is near. Without resource-augmenting technical change, the backstop will eventually indeed become competitive as the effective resource price continues growing. Therefore, the economy will have depleted the resource stock and switches from the resource to the substitute at point $\mathrm{D}$, implying that expectations again are truly rational along the path leading to the implementation of the backstop technology.

During the transition, the economy cannot be located in the shaded part of the figure, because in that area $\theta_{E}>\theta_{H}^{*}$ holds, implying that the backstop will be used instead of the resource. It is clear from the figure that if $Z \geq Z_{1}$, each value of the stock is associated with

\footnotetext{
${ }^{29}$ Cf. Figure III of Krugman (1991, p. 661) and Figure V of Matsuyama (1991, p. 646).
} 
two equilibrium values for $\theta_{E}$. This region is the 'overlap', in the terminology of Krugman (1991): in this region, self-fulfilling expectations can lead the economy to either of the two steady state equilibria. If $Z<Z_{1}$, however, the unique equilibrium path is the one leading to the backstop steady state. Hence, in panel (a) of Figure 5, multiple equilibria exist as long as the initial stock is large enough: $Z>Z^{*}$ is a sufficient condition for overlap. This, however, is not a general result. The overlap for $Z>Z^{*}$ disappears if the productivity of the backstop technology, measured by $\eta$, becomes small, as is shown in panel (b) of the figure. For $Z>Z_{2}$, the path leading to the backstop steady state is no longer an equilibrium. For $Z<Z_{1}$, the path towards the fossil steady state is not an equilibrium, because it would imply $\theta_{E}>\theta_{H}^{*}$ (in the shaded part of the diagram). For values of the stock in between $Z_{1}$ and $Z_{2}$, the path leading to either steady state can be chosen. Moreover, for values of the stock in between $Z_{0}$ and $Z_{2}$, each value of $Z$ is associated with two values of $\theta_{E}$ on the equilibrium path leading to the backstop technology: one with a relatively high income share (and high resource-augmenting technical change resulting in an increasing $Z$ over time) and one with a relatively low resource income share (and low resource-augmenting technical change resulting in a decreasing $Z$ over time). ${ }^{30}$ The fact that the overlap is now located to the left of the fossil steady state $\left(Z_{0}<Z_{1}<Z_{2}<Z^{*}\right.$ ) implies that the backstop steady state cannot be reached if the economy starts with an initial stock satisfying $Z(0)>Z^{*}$.

Figure 6: Phase diagrams - Unique equilibria

(a) Unique transition to backstop

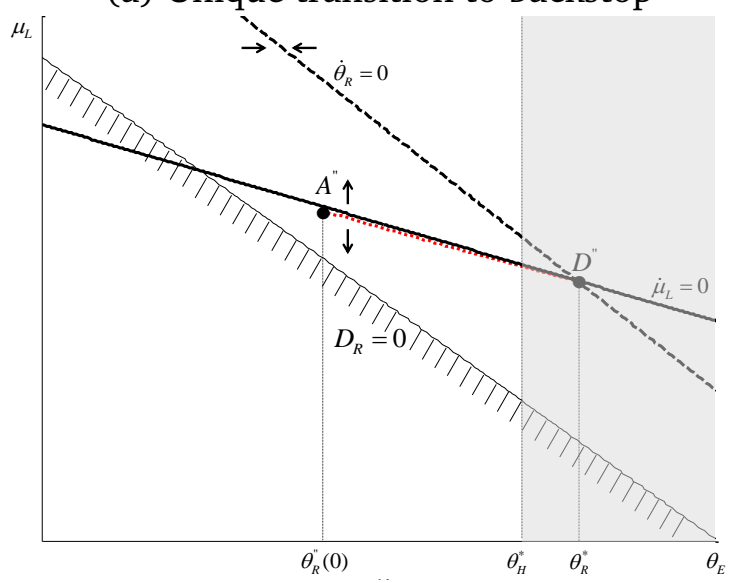

(b) Unique fossil equilibrium if $Z(0)>Z^{*}$

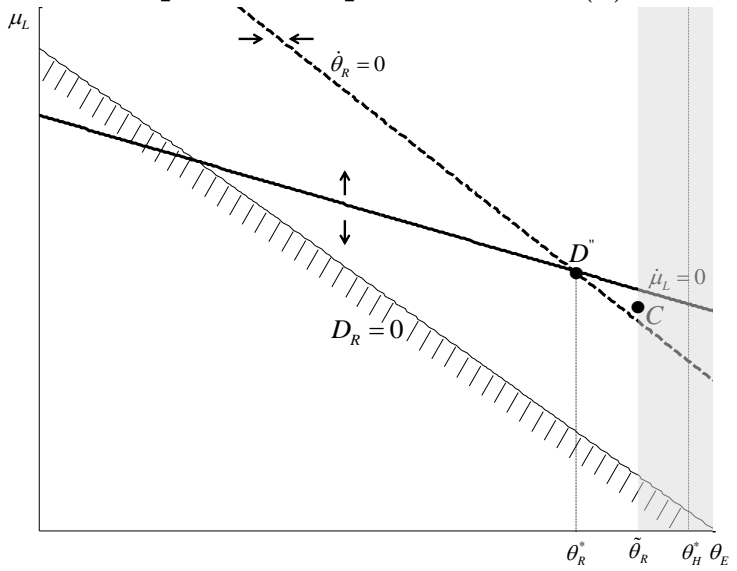

Notes: In panel (a), the transition along the dotted path from point $\mathrm{A}^{\prime \prime}$ towards the fossil steady state at point $\mathrm{D}^{\prime \prime}$ cannot be an equilibrium, because $\theta_{R}>\theta_{H}^{*}$ in the grey part of the phase diagram. This would imply fossil energy use while the backstop technology is cheaper. In panel (b), the transition towards the backstop technology cannot be an equilibrium if $Z(0)>Z^{*}$ (implying $\theta_{R}(0)<\theta_{R}^{*}$ ), because point $\mathrm{C}$ (the point at which resource-augmenting technical change vanishes) cannot be reached from the left.

\footnotetext{
${ }^{30}$ Hence, using the terminology of Benhabib and Perli (1994), our model both features local indeterminacy (e.g., for $Z_{0} \leq Z \leq Z_{2}$ in panel (b) of Figure 5, where multiple values of the non-predetermined variable $\theta_{E}$ on the path leading to the same steady state are associated with each value of the predetermined variable $Z$ ) and global indetermincay (e.g., for $Z>Z_{1}$ in panel (a) and $Z_{1} \leq Z \leq Z_{2}$ in panel (b), where multiple values of $\theta_{E}$ on paths leading to different steady state equilibria are associated with each value of $Z$ ).
} 
It is interesting to know under which conditions the overlap covers the interval $Z>Z^{*}$. Although, as Krugman (1991, p. 664) states, determining explicitly the boundaries of the overlap is "an algebraic nightmare", even in the linear model that Krugman discusses, with the help of Figure 3 we can transparently discuss how changes in parameters make the multiplicity of equilibria for $Z>Z^{*}$ more likely to arise. The productivity in the backstop sector, $\eta$, is an interesting parameter to consider in this context. The energy income share in the backstop regime depends negatively on this parameter. Hence, the first result in Proposition 1 implies that the transition to the backstop technology is the unique equilibrium if $\eta$ is large enough to get $\theta_{H}^{*}<\theta_{R}^{*}$. An example of such a case without an overlap is shown in panel (a) of Figure 6, where the path leading to the fossil steady state at point $\mathrm{D}^{\prime \prime}$ can be excluded as an equilibrium, because in the grey part of the figure the backstop is cheaper than the fossil energy source.

In the Figures 3-4 we depicted the situation with $\theta_{H}^{*}>\theta_{R}^{*}$ in which an overlap exists. Starting in the backstop steady state and working backwards in time, we have constructed the equilibrium path and found that it starts from a low resource share, i.e., point $\mathrm{A}$ is to the left of point $\mathrm{C}$ in Figure 3. This naturally happens if the gap between $\theta_{R}^{*}$ and $\theta_{H}^{*}$ is small, i.e., if $\eta$ is relatively large. However, for smaller values of $\eta$, the gap between $\theta_{R}^{*}$ and $\theta_{H}^{*}$ increases and point $C$ shifts to the right. If $\eta$ is small enough, point $C$ will be located to the right (above) the instable arm of the dynamical system in Lemma 1 and the path leading to point $\mathrm{C}$ with both types of innovation extends to the right of $\theta_{H}^{*}$, instead of to the left of $\theta_{R}^{*}$. In this case there cannot be an equilibrium path to the backstop steady state if the initial stock $Z(0)$ exceeds the fossil steady state value $Z^{*}$. This is intuitive as well: a high resource income share (corresponding with a high resource price) implies low resource demand during the transition to the backstop steady state. As a result, if the resource is abundant, cumulative resource use falls short of the resource stock, which cannot occur in equilibrium. An example of this case is shown in panels (b) of Figures 5 and 6, where the path to the backstop steady state does not extend to the region with $Z>Z^{*}$, and where point $\mathrm{C}$ cannot be approached from the left, respectively.

\subsection{Why self-fullfilling prophecies may arise}

The explanation for the existence of an overlap region with multiple equilibria can be found in the seminal contribution by Cooper and John (1988), which was applied in various dynamic contexts in Murphy et al. (1989), Krugman (1991), and Benhabib and Perli (1994): there is a strategic complementarity in investment because the returns to investment by one (type of) investor increases with investment by another (type of) investor. In particular, the complementarity exists between investment in resource-augmenting technologies (increasing the 
future knowledge stock $Q_{R}$ ) and investment in resource conservation (increasing the future resource stock $S$ ). If there is more investment in resource-augmenting technologies, the backstop remains uncompetitive for a longer period and future fossil demand will be higher thus increasing the returns to fossil conservation. Vice versa, if resource owners conserve more of the fossil stock for future use, the current price of energy services increases and makes investment in fossil saving more profitable. The result is that without the expectation of a backstop becoming competitive in the future both groups tend to invest relatively more which justifies each other's high investment level as well as the expectation, while with the expectation of a competitive backstop both groups invest little, which also justifies the expectation and each other's investment. ${ }^{31}$

More formally tied to the math and the phase diagrams, the resource share $\theta_{R}$ affects the two types of investment in the same direction. First, the value of investment in resource-augmenting technologies increases with the future resource share, since this value is measured by $\lambda_{R}$, which is solved from equations (7c) and (8) as

$$
\lambda_{R}(t)=\int_{t}^{\infty} \theta_{R}(\tau) \beta(1-\beta)\left[Y(\tau) p_{Y}(\tau) / Q_{R}(\tau)\right] e^{\int_{t}^{\tau} r(s) d s} d \tau .
$$

Second, the value of investments in the future resource stock depend on future resource prices which are positively linked to the resource share. In comparison with the transition path towards the fossil steady state, the initial resource share is lower on a transition path to the backstop steady state since the same amount of fossil is sold in a shorter period, making resources more abundant and lowering its price (cf. Figure 5). The lower resource share discourages both investments, thus making them complements.

When expectations select the equilibrium path, there is no guarantee that firms select the path that gives them highest profits. Yet, on a rational expectations equilibrium paths, no individual agent has an incentive to deviate from the equilibrium choice. Given equilibrium prices and choices of all other agents, atomistic agents do not perceive benefits from deviating and may end up with suboptimal profits. Of course, if all agents coordinate and collectively change behavior, the equilibrium might shift to the path with higher profits. However, coordination among many investors is difficult.

At an equilibrium path, individual investors are indifferent between investing in the different types of $R \& D$ and resource conservation. If, starting from an equilibrium, an individual investor increases the share of investment in resource-augmenting technologies compared to labor-

\footnotetext{
${ }^{31}$ Because the technology of the backstop is fixed and no investment takes place in backstop technologies, there cannot be strategic complementarity between innovators in the fossil sector and innovators in the backstop sector; however, resource owners choose to either conserve resources or extract fast and this is the investment choice that affects (future) demand for innovations in the fossil sector and generates the complementarity.
} 
augmenting technologies in his portfolio, equation (9) implies that - given the equilibrium prices on other markets and the expectations and investment strategies of other investors the return to investment in resource-augmenting technologies goes down relative to the return to labor-augmenting technologies and resource conservation, and vice versa, which confirms that the individual innovator/investor has no incentive to deviate. Similarly, if, starting from an equilibrium, an individual investor invests more in resource conservation by selling less of it today, equation (5a) implies that - given the equilibrium prices on other markets and the expectations and investment strategies of other investors - the current resource price goes up compared to future resource prices, implying that the return to resource conservation goes down relative to the rate of return to $\mathrm{R} \& \mathrm{D}$, and vice versa. Hence, individual investors have no incentive to deviate from an equilibrium.

The strategic complementarity between investments undertaken by atomic investors creates the possibility of multiple equilibrium steady states (global indeterminacy) and multiple equilibrium transition paths towards a given steady state (local indeterminacy, cf. Benhabib and Perli (1994)). The implication for the energy transition is, first, that whether the economy is locked into fossil or not might depend on expectations and, second, that even if fossil is abandoned, there might be a fast transition or a slow transition, again depending on expectations. The red path in panel (b) of Figure 5 illustrates this possibility: if $Z_{1}<Z(0)<Z_{2}$, the initial condition is consistent with two starting values for the resource share, implying that there is a rational expectation equilibrium with a fast transition - with low resource prices, low initial resource share, and monotonic depletion of the effective resource stock - as well as one with a slow transition - with high resource prices, high resource share, and prolonged resource-augmenting technical change that increases the effective resource stock. The strategic complementarity between resource conservation and investment in resource-augmenting technologies drives both global and local indeterminacy.

Strategic complementarity is not a sufficient condition. The complementarity needs to be so strong that coordination of investment can overrule the inherent cost advantage of the backstop or resource, where the former is measured by (the inverse of) cost parameter $\eta$ and the latter by effective resource abundance $Z$. As shown in panels (b) of Figure 5 and 6 a higher cost of the backstop and a greater abundance of fossil reserves (and thus a low initial resource income share) make multiplicity less likely. Under these conditions, during a transition to the backstop technology the energy income share will eventually become really high, implying that future incentives for resource-saving technical change will be so strong that investors cannot rationally believe that the transition will be made to the backstop and no coordination failure can arise. 


\section{Numerical illustration}

In this section, we quantify the results of the model by performing a numerical analysis. ${ }^{32}$ The aim of this exercise is to show in which scenarios the introduction of the backstop technology actually is a self-fulfilling prophecy and to highlight the differences between the two equilibria in terms of the time profiles of fossil fuel use and resource-augmenting technical progress.

We first calibrate the model to match data on energy income shares and consumption growth in modern industrialized economies. For the elasticity of substitution between labor and resource services, we choose $\sigma=0.45$, which is within the range of 0.17 to 0.61 that Van der Werf (2008) reports. The parameter $\beta$ is the output elasticity of the primary factors, labor and fossil fuel, in both service sectors. Our value of 0.8 lies within the range of the labor income shares reported in Gollin (2002) and is in line with the average share of fossil fuel consumption in total energy consumption in the OECD countries, which amounted to 82 percent over the years 2000-2011 (World Bank, 2014). We set the rate of pure time preference $\rho$ to 0.01 and choose $\gamma=0.50$ for the final good production function parameter. The backstop productivity parameter $\eta$ is fixed at 10 , so that $\theta_{H}^{*}=0.21$, whereas $\theta_{R}^{*}=0.19$. The initial stocks of quality in both sectors $Q_{L 0}, Q_{R 0}$ are normalized to unity. We choose an initial non-renewable resource stock of 300 to obtain an initial energy income share of 7.8 and 9.9 percent in the scenarios with and without the transition to the backstop technology, respectively, which is in line with the average energy expenditure share in GDP of 8.8 percent over the period 1970-2009 in the United States (U.S. Energy Information Administration, 2011). In the scenarios with and without the transition to the backstop technology, our implied initial reserve-to-extraction rates are 105 and 147, respectively. The implied initial price of the backstop technology compared to the resource price, $p_{Y H}(0) / p_{Y R}(0)$, amounts to 5.9 and 3.8 in the two scenarios. We fix the labor supply $L^{S}$ at 0.14 and the research productivity parameter $\xi_{R}$ at 20/3 to obtain an initial yearly consumption growth rate of 1.7 percent in both scenarios, which is equal to the average yearly growth rate of GDP per capita in the United States over the period 1970-2011 (The Conference Board, 2011). Our initial stock values imply $Z(0)=2143$, whereas $Z^{*}=531$, yielding $Z(0)>Z^{*}$.

In our benchmark calibration, $Z(0)$ is located within the overlap region. Figure 7 shows the time profiles for fossil fuel use (panel a) and resource-augmenting technical change (panel b) for the two equilibria. If the backstop technology is expected not to become competitive (see the dotted lines), resource extraction starts out relatively low and is monotonically declining over time. Resource-augmenting technical change is relatively high and monotonically increasing

\footnotetext{
${ }^{32}$ For the numerical simulation, we use the relaxation algorithm explained in Trimborn et al. (2008). Appendix A. 4 provides details about the solution procedure that we have used.
} 
(a) Fossil fuel use

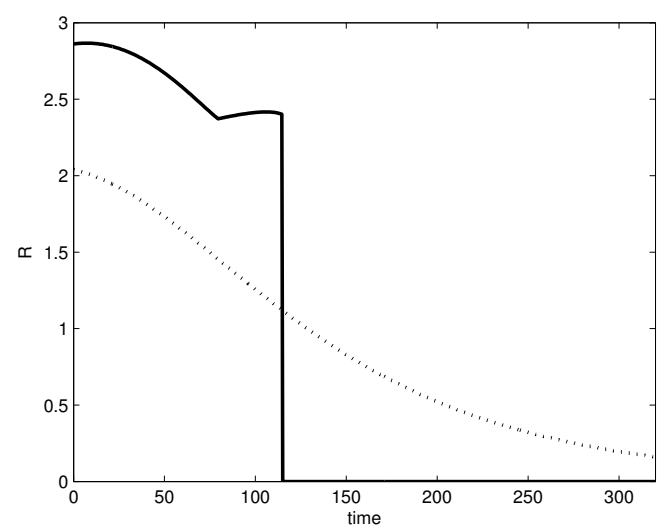

(b) Resource-augmenting research

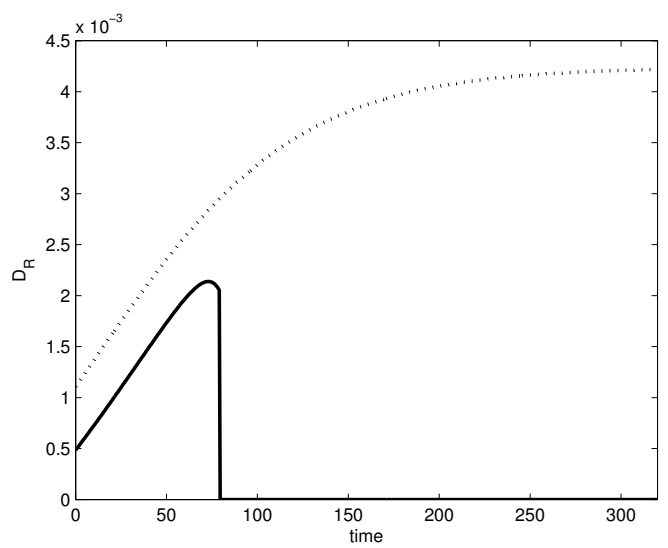

Notes: The solid line represents the transition to the backstop technology, whereas the dotted line shows the scenario in which the backstop technology does not become competitive. Parameters and initial stocks are set at their benchmark values.

over time. On the contrary, if the future implementation of the backstop technology is expected (see the solid lines), current resource extraction is relatively high, whereas resource-augmenting technical change is modest and drops to zero before the backstop technology actually becomes competitive. The kink in the resource extraction path is due to this drop in resource-augmenting technical change.

Figure 8: Region with multiple equilibria

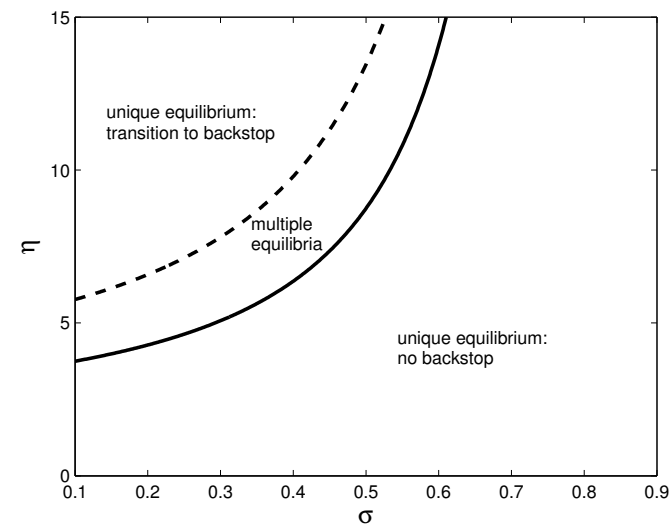

Notes: Above the dashed line, the transition to the backstop technology always takes place. Below the solid line, the transition never takes place. In between the two lines, multiple equilibria exist so that the future implementation of the backstop technology is a self-fulfilling prophecy. Parameters and initial knowledge stocks are set at their benchmark values. The initial resource stock is chosen large enough to get $Z(0)>Z^{*}$.

The elasticity of substitution $\sigma$ is a measure for how difficult it is to replace fossil fuels by conventional factors of production, whereas $\eta$ measures the productivity of a non-conventional substitute for fossil fuels. Both parameters are important for the characteristics of the energy transition. Figure 8 shows for which combinations of those two parameters there exist multiple 
equilibria in the model.

For all points above the dashed line in the figure, the backstop technology is relatively productive, so that it will always become competitive in the future. The dashed line is upwardsloping, because an increase in the elasticity of factor substitution increases the energy income share in the backstop economy, so that a higher productivity is needed to guarantee the implementation of the backstop technology. For all points below the solid line, the productivity of the backstop technology is so low that it will never become competitive. ${ }^{33}$ The area in between the broken line and the dashed line gives the combinations for which the implementation of the backstop technology is a self-fulfilling prophecy.

\section{Welfare analysis}

The aim of this section is twofold. First, we want to compare the decentralized market outcome with the optimum. Second, we want to compare welfare in an economy that experiences a transition to the backstop technology with welfare in an economy in which the backstop technology is never introduced. In order to make these comparisons, we will first discuss the social optimum.

\subsection{Social optimum}

The social planner maximizes lifetime utility $U(t)=\int_{t}^{\infty} \ln C(z) e^{-\rho(z-t)} d z$, subject to (1), (3a), (3b), (4), (12), (6), (17) and non-negativity constraints on employment and energy inputs. The full solution to the problem of the social planner is derived in Appendix A.2. Here we stick to a discussion of the first-order conditions with respect to $x_{i k}, D_{i k}$, and $q_{i k}$, which uncover the differences between the market equilibrium and the social optimum. Those three first-order conditions can be written as

$$
\begin{aligned}
p_{x i k} & =q_{i k} p_{Y}, \\
\lambda_{i k} \xi_{i} Q_{i} & \leqq w_{D} \quad \text { with equality if } D_{i k}>0, \\
-\xi_{i} D_{i k}+\frac{\beta}{1-\beta} x_{i k} p_{Y} & =-\dot{\lambda}_{i k}+\rho \lambda_{i k} .
\end{aligned}
$$

There are two differences between the social optimum and the market equilibrium. When comparing (7a) and (28a), it is clear that the market equilibrium has an additional factor $1 /(1-$ $\beta)>1$ on the right-hand side. The reason is the imperfect competition in the intermediate

\footnotetext{
${ }^{33}$ The dashed line represents the equality $\theta_{R}^{*}=\theta_{H}^{*}$, cf. Proposition 1, part ii. The numerical construction of the solid line uses the fact that, because $Z(0)>Z^{*}$, the tranistion to the backstop cannot be an equilibrium outcome if the overlap does not cover the interval $Z>Z^{*}$.
} 
goods sector: due to monopolistic competition, intermediate goods producers charge a markup over their marginal production costs. The second difference is the additional term $-\xi_{i} D_{i k}$ on the left-hand side of (28c) compared to (7c). The reason for this discrepancy is the existence of knowledge spillovers within service sectors: individual intermediate goods producers ignore the effect of their R\&D on the productivity of researchers in the other firms within the same service sector, implying that this specific term drops out in the description of the market equilibrium.

\subsection{Social optimum versus market equilibrium}

The derivation of the social optimum in Appendix A.2 shows that, similar to the decentralized equilibrium, the problem of the social planner may also have multiple candidate solutions that satisfy the first-order and transversality conditions. In the social optimum, the candidate solution(s) with highest welfare constitute(s) the solution. The region within the solid and the

Figure 9: Planner solution - Region with multiple candidates

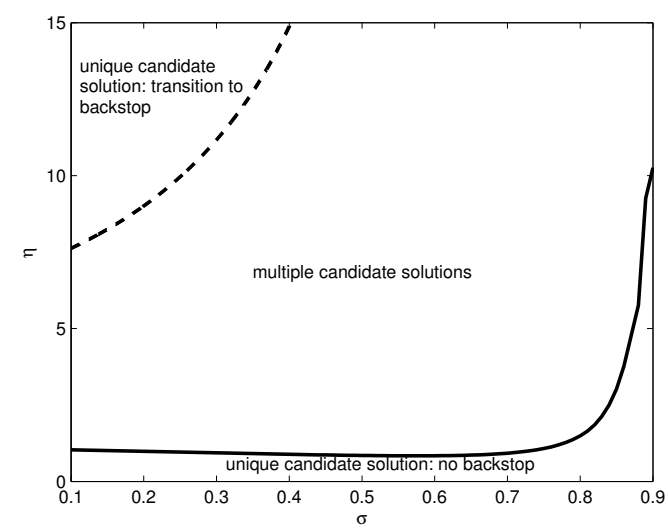

Notes: Above the dashed line, transition to the backstop technology is the only candidate for an optimum. Below the solid line, the transition to the fossil steady state is the only candidate for an optimum. In between, the transition to the backstop technology and to the fossil steady state are both candidates for an optimum. The initial resource stock is chosen large enough to get $Z(0)>Z^{*}$.

dashed line in Figure 9 gives combinations of the backstop technology productivity parameter $\eta$ and the elasticity of factor substitution $\sigma$ for which multiple candidate solutions exist: one with and one without the eventual implementation of the backstop technology. For all points above the dashed line, the backstop technology is relatively productive and there is no candidate solution without the implementation of the backstop technology. For all points below the solid line, the productivity of the backstop technology is so low that there is no candidate solution with the implementation of the backstop technology. ${ }^{34}$

A comparison of Figures 8 and 9 reveals that in the decentralized market equilibrium, the dashed line is located lower, whereas the solid line is located higher in the $(\sigma, \eta)$ plane.

\footnotetext{
${ }^{34}$ The dashed line is directly derived from the condition $\xi_{R}=(1-\gamma)^{-\sigma} \eta^{1-\sigma}$. The solid line is determined numerically. Details can be found in Appendix A.2.
} 
Intuitively, the lower position of the dashed line can be explained as follows: along the path that leads to the fossil steady state, the value of knowledge spillovers in the resource sector is relatively large compared to the spillovers in the labor sector, because of the finite availability of the resource. As a result, the market invests relatively too little in resource-saving technical change, so that the range of backstop productivity parameter values for which the market always shifts to the backstop becomes larger. Hence, the dashed line is located lower in the decentralized equilibrium than in the optimum. Similarly, along the path that leads to the implementation of the backstop technology, the value of the knowledge spillovers in the resource sector are relatively small compared to spillovers in the labor sector, because accumulated knowledge in the resource sector becomes worthless from the time of the shift to the backstop technology onwards. As a result, the market relatively overinvests in resource-saving technical change, so that the range of backstop productivity parameter values for which the market never shifts to the backstop technology becomes larger. Hence, the solid line is located relatively higher in the decentralized equilibrium. It follows that the multiple candidate solutions region in the centralized economy is larger than the multiple equilibria region in the market economy.

Figure 10: Welfare difference between backstop and fossil

(a) Optimum

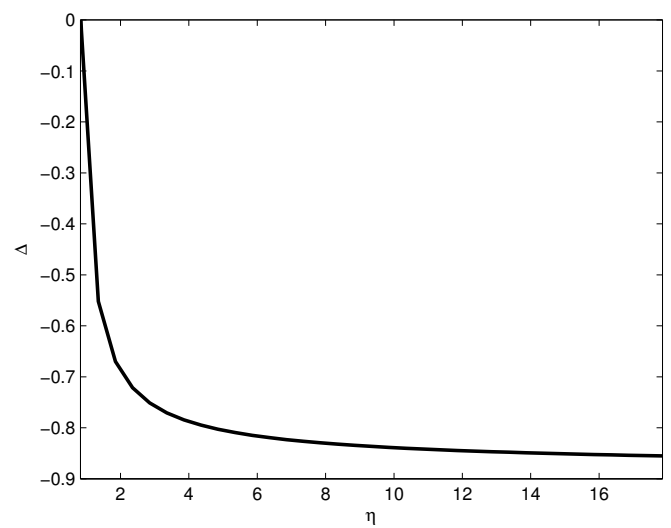

(b) Market

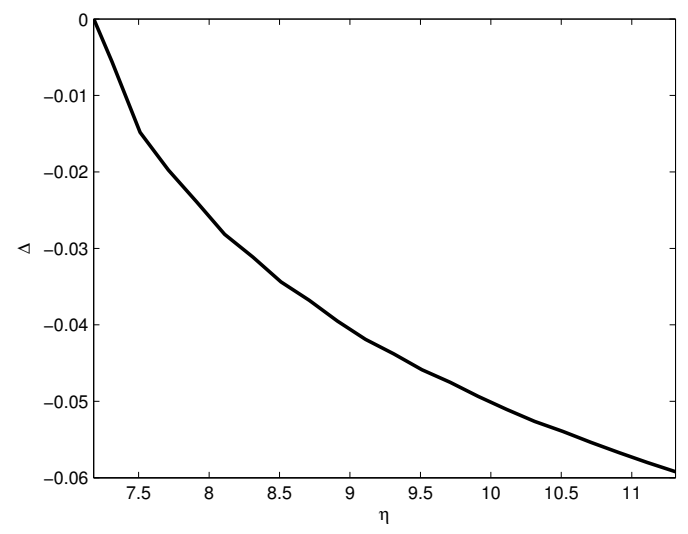

Notes: The welfare difference measure $\Delta$ gives the relative change in initial consumption along the backstop path that would give the same change in welfare as when the economy would move from the backstop to the fossil transitional path. The interval for $\eta$ in each panel corresponds with the distance between the dashed and solid lines evaluated at at $\sigma=0.45$ in Figures 8 and 9 , respectively. Parameters and initial stocks are set at their benchmark values.

Figure 10 shows the change in welfare if the economy would move from the backstop transitional path to the fossil transitional path, both for the social planner problem (panel (a)) and the decentralized market equilibrium (panel (b)). The welfare difference measure $\Delta$ in the figure is defined as the relative change in initial consumption along the backstop path, while keeping the consumption growth rate unchanged, that would give the same change in welfare as when the economy would move from the backstop to the fossil transitional path. For example, $\Delta=-0.05$ means that moving from the backstop equilibrium to the fossil equilibrium gives 
a welfare loss equivalent to a downward shift of the consumption time profile by 5 percent. ${ }^{35}$ The two panels show the interval of the backstop productivity parameter $\eta$ for which the social planner problem has two candidate solutions and the market economy has two equilibria, where all other parameters are set at their benchmark values. Hence, the intervals for $\eta$ in panels (a) and (b) correspond with the vertical distance between the dashed and solid lines in Figures 9 and 8, respectively, at $\sigma=0.45$.

Panel (a) shows that at the lowest $\eta$ for which there are two candidate solutions, the welfare generated by the path heading towards the fossil steady state is equal to the welfare generated by the transition to the backstop technology (i.e., $\Delta=0$ ). For higher productivity values, the transition to the backstop technology delivers a strictly higher level of welfare (i.e., $\Delta<0$ ). Therefore, in this interval the social planner will select the path leading to the introduction of the backstop technology. Panel (b) shows a comparable result for the decentralized market economy: at the lowest $\eta$ for which there exist multiple equilibria, the welfare generated by the path leading to the fossil steady state is equal to the welfare generated by the transition to the backstop technology. If the backstop productivity exceeds this threshold level, the transition to the backstop technology gives strictly higher welfare. It was already clear from Figures 8 and 9 that the lowest $\eta$ for which there are multiple equilibria in the decentralized equilibrium is higher than the minimum value for $\eta$ that is required for multiple candidate solutions in the social planner problem.

Summarizing the results in this section, we have learned from the welfare analysis that the regions in $(\sigma, \eta)$-space for which the backstop will never or always be implemented (the areas below and above the solid lines in Figures 8-9, respectively) are smaller in the centralized economy than in the market economy, because the market generates too much (not enough) resource-saving technical progress along the transitional path towards the backstop (fossil) steady state. Moreover, the welfare analysis has shown that if there exist multiple equilibria in the benchmark market economy, the equilibrium leading to the implementation of the backstop technology generates (at least weakly) higher welfare.

\subsection{Extension: climate change}

So far, we have ignored the effects of climate change. In this subsection we introduce damages from the stock of accumulated carbon in the atmosphere. We consider the simplest case of linear damages in the utility function and zero depreciation of atmospheric carbon, implying that the atmospheric carbon stock $A=S_{0}-S$ develops according to $\dot{A}=-\dot{S}=R$, and the utility function of the social planner changes to $U(t)=\int_{t}^{\infty}\left(\ln C-\psi\left(S_{0}-S\right)\right) e^{-\rho(z-t)} d z$, where $\psi$ is

\footnotetext{
${ }^{35}$ Technically, our welfare measure reads $\Delta \equiv e^{-\rho\left(\Lambda_{H}-\Lambda_{F}\right)}-1$, where $\Lambda_{H}$ and $\Lambda_{F}$ are the welfare levels corresponding to the backstop and fossil transitional paths, respectively. Details can be found in Appendix A.3.
} 
the constant instantaneous marginal damage of a unit of atmospheric carbon in utility terms. ${ }^{36}$ The solution to the problem of the social planner is derived in Appendix A.2.7. Here, we present the results of the simulation analysis in order to highlight the consequences of climate change for the desirability of the transition to the backstop.

We impose $\psi=0.001$, which corresponds with a marginal damage of about 140 US\$ per ton carbon with $\rho=0.01$, implying for the resource expenditure share (which is now defined to include climate costs) $\theta_{R}(0)<\theta_{R}^{*}$, as in the model without climate damage. ${ }^{37}$ Along the transitional path to the backstop technology in our benchmark simulation, the scarcity rent equals zero, implying that cumulative extraction is smaller than $S_{0}$. Intuitively, society wishes to leave part of the fossil fuel stock untapped to avoid serious climate damage, and switch to the backstop technology before full depletion.

Figure 11: Climate change - Region with multiple candidates

(a) $\rho=0.01$

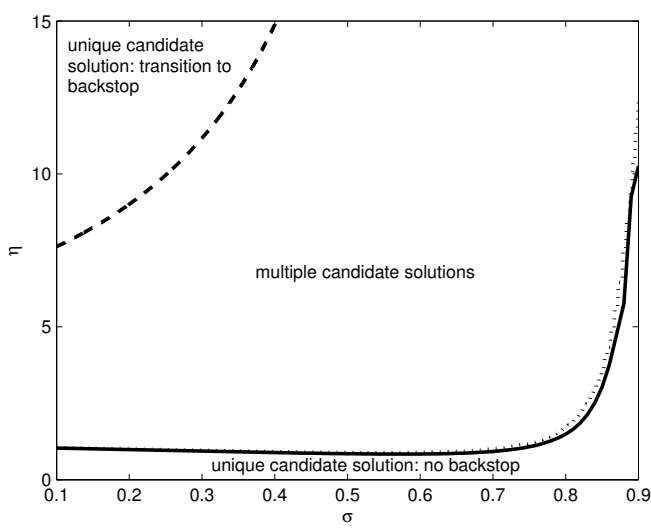

(b) $\rho=0.05$

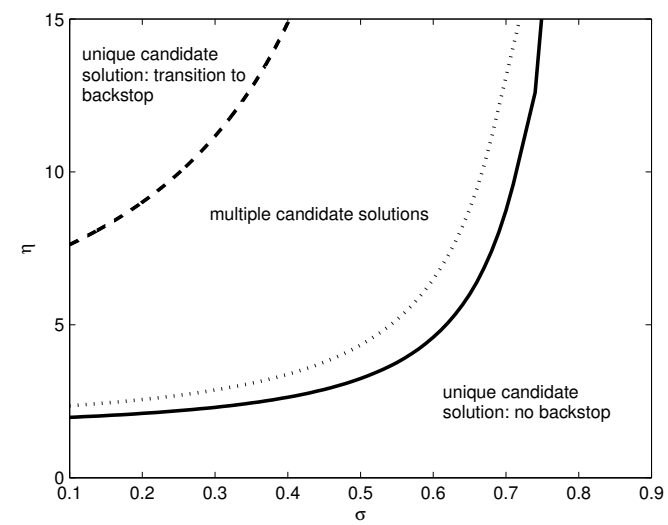

Notes: Above the dashed lines, transition to the backstop technology is the only candidate for an optimum. Below the solid (dotted) lines, the transition to the fossil steady state is the only candidate for an optimum in the model without (with) climate damages. In between, the transition to the backstop technology and to the fossil steady state are both candidates for an optimum. Panel (a) shows the case with $\rho=0.01$ and panel (b) with $\rho=0.05$. In all cases in the figure, we have $Z(0)>Z *$, as in the model without climate damage.

Along the path to the fossil steady state as well as along the path to the backstop technology, welfare is lower than in the case without climate damages. On the one hand, the negative welfare effect is stronger in case of a transition to the backstop technology, because of a 'Green Paradox' effect (cf. Sinn, 2008; Van der Ploeg and Withagen, 2015): along the path to the backstop technology, initial extraction is larger than along the path towards the fossil steady state, meaning 'front-loading' of resource extraction and carbon emissions. Due to discounting,

\footnotetext{
${ }^{36}$ We have chosen units such that a unit of resource use results in a unit of carbon emissions.

${ }^{37}$ We take a global output level of 75 trillion US\$, corresponding with global output in 2013 (World Bank, 2014), and a carbon stock of 12,879 Gigaton, corresponding with the probable oil, natural gas, and coal reserves reported in Edenhofer and Kalkuhl (2009). In our benchmark case with $\beta=0.8, \rho=0.01$, and $S_{0}=300$, we get $Y=0.10$. Let resource units correspond with tonnes of carbon. Then we find a marginal damage of about 140 US\$ per ton carbon.
} 
this front-loading implies that the present value of climate damage is larger. As a result, the introduction of climate damages decreases welfare of the path leading tot the backstop technology further than welfare of the path leading to the fossil steady state. On the other hand, the switch to the backstop technology reduces cumulative resource extraction, because part of the resource stock remains untapped, as in Van der Ploeg and Withagen (2014). If the marginal damage of carbon emissions is large enough, the social planner will even decide to leave the entire initial resource stock in situ and use the backstop technology from the beginning. Having opposite effects on climate damage during the transition to the backstop technology, these 'Green Paradox' and the 'cumulative extraction' effects are likely to have counteracting implications for the different regions in Figure 9.

Figure 11 shows the net effect of climate damages on the different regions in the benchmark scenario. In our simulation, the 'Green Paradox' effect dominates the 'cumulative extraction' effect, implying that the region for which society does not want to use the backstop in Figure 11 is larger than in Figure 9. The frontier of this region is indicated by a dotted (solid) line for the model with (without) climate damage. The difference between the situation with and without climate damage is more pronounced in panel (b) than in panel (a) of Figure 11, because due to the higher discount rate, $\rho=0.05$ instead of $\rho=0.01$, the Green Paradox effect is more important in the former case than in the latter. Because steady-state income shares $\theta_{R}^{*}$ and $\theta_{H}^{*}$ are not affected, the 'unique candidate solution: transition to backstop' region remains unchanged. ${ }^{38}$

Figure 12: Climate change - Welfare difference between backstop and fossil

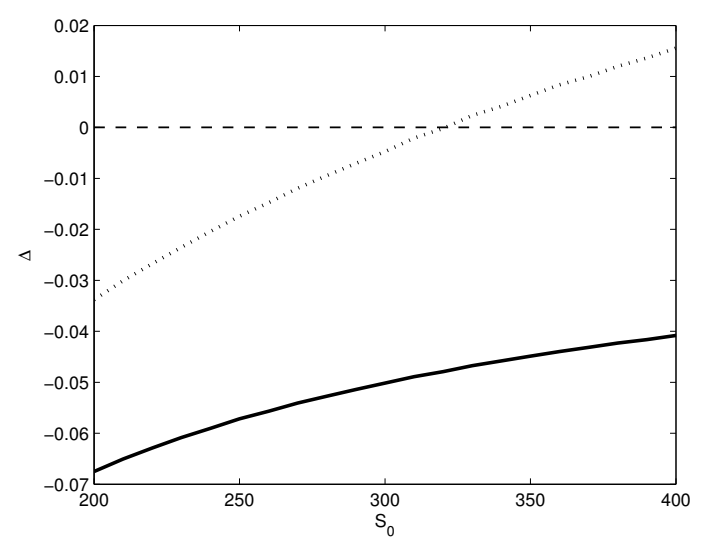

Notes: The welfare difference measure $\Delta$ gives the relative change in initial consumption along the backstop path that would give the same change in welfare as when the economy would move from the backstop to the fossil transitional path in the market equilibrium without climate damage (solid line) and with climate damage (dotted line). Parameters are set at their benchmark values.

\footnotetext{
${ }^{38}$ In order to show what happens if the 'cumulative extraction' effect dominates, we would need to explore in detail the case in which $Z(0)<Z^{*}$, which would complicate the exposition considerably. Therefore, we leave this case for future research.
} 
In the laissez faire market equilibrium, the fossil fuel stock will be fully depleted along the transitional path towards the backstop technology. As a result, only the Green Paradox effect of the transition to the backstop is present there. Figure 12 shows the change in welfare if the economy would move from the backstop transitional path to the fossil transitional path, both for the case without climate damage (solid line) and with climate damage (dotted line). We again use a measure of equivalent relative changes in consumption, $\Delta$, as defined in footnote 35 , for the welfare differences. The figure shows that the higher the initial fossil fuel stock $S_{0}$, the smaller is the welfare loss of moving from the backstop to the fossil equilibrium. For our benchmark value of $S_{0}=300$, the backstop path still generates higher welfare than the fossil path, despite the higher present value of climate damages (see the dotted line). However, for larger values of the initial resource stock, the market economy is better off without backstop due to the Green Paradox effect.

\section{Conclusion}

This paper has investigated the interaction between innovation and the energy transition from fossil fuels to renewable energy. For this purpose, we have constructed a growth model with a non-renewable resource and a backstop technology in which profit incentives determine both the rate and the direction of technical change endogenously. We take into account that natural resources and man-made factors of production are poor substitutes, that energy generation with the backstop technology is costly, and that resource-augmenting technologies become worthless after a shift from fossil fuels to renewable energy.

Our main finding is that the future large-scale implementation of renewable energy might be a self-fulfilling prophecy, due to the existence of strategic complementarity between the decisions of investors. If it is expected that backstop technologies will become competitive eventually, fossil resource owners want to sell their resources before the introduction of the backstop and the market for resource-saving inventions will be small, so that incentives for resource conservation and investment in resource-augmenting technologies will be eroded and renewable energy will indeed become competitive in the long run. Conversely, if investors expect that backstop technologies will not be able to produce renewable energy at competitive prices on a large scale, fossil resource depletion will be more conservatively and the market for resource-saving inventions will become significant; as a result renewable energy will indeed be relatively unattractive in the long run. Only when the backstop is sufficiently cheap, the transition to renewable energy will always take place in a decentralized market economy.

The existence of expectations-driven multiple equilibria that can be Pareto-ranked has important implications for policy. As is standard in other models of directed technical change, our 
model includes externalities that can be addressed by policies: the benefits from research are not fully appropriated and there is monopolistic competition. However, if the coordination of expectations is difficult, additional temporary policies might be needed to steer the economy into the direction of the optimal path. Our welfare analysis suggests that in case of multiple decentralized equilibria, the transition to the backstop technology generates higher utility. The real challenge for policy design is then to know if the market is right or wrong, in the sense of choosing the equilibrium with relatively higher or lower welfare level, when it bets on fossil fuels: if we would know that we are in the multiple equilibria range and the market expects the economy to use fossil forever, the development of resource-augmenting technologies needs to be discouraged (to steer away expectations). However, if we are in the range in which using fossil fuels forever is optimal, we need to subsidize resource-augmenting R\&D (because of spillovers).

An interesting extension of our model would be the introduction of climate damages from the use of fossil fuels. As a first step in this direction, in Section 5.3 we have considered the simplest case of linear climate damages resulting from accumulated carbon emissions. In our simulation, we found that for certain parameter combinations the backstop is no longer introduced in the optimum with climate change while it was optimally introduced in the absence of climate damages. The reason is that conservation of fossil for the future is no longer needed if the backstop is ultimately introduced, which implies a higher concentration of emission damages in earlier stages as compared to a fossil-forever solution. This 'Green Paradox' result can be strong enough to revert the result derived without climate damage that the market economy is best off with the backstop.

Besides the two equilibria that we have characterized, the 'fossil forever' and the transition from fossil to the backstop, it is possible that there are parameter combinations for which equilibria exist that start with a temporary backstop regime before resource extraction takes place. Such a temporary initial backstop regime would require a resource price that grows at a faster rate than the rate of interest, ensuring that resource owners initially prefer to postpone extraction. It seems interesting to investigate under which conditions such a regime can actually exist.

Furthermore, it would be interesting to analyze aspects of technical change that matter for the transition but are left out in our model. In particular, we have abstracted from technology spillovers between sectors and from technical change in the backstop sector. One would expect that energy sectors could learn from innovations in the rest of the economy, and - maybe to a lesser degree - also the other way around. Two new mechanisms will become relevant. First, innovation effort in one sector affects technology in the other sector, so that spillovers are associated with flow variables $D_{L}$ and $D_{R}$. This affects the wedge between social and 
private returns to innovation, but does not necessarily affect the coexistence of a fossil-only steady state and a backstop steady state. ${ }^{39}$ Second, the productivity of investment in innovation might depend on a measure of the aggregate knowledge stock, so that spillovers are associated with both stocks of knowledge as in the 'limited state dependence' case of Acemoglu (2002) or in the generalized case of Hart (2012). Depending on the exact modeling, this second mechanism might make resource-augmenting technical change increasingly costly relative to labor-augmenting technical change when the relative stock of resource-augmenting technologies grows. If strong enough, this force would tend to raise the fossil energy share and thus make the backstop introduction inavoidable. ${ }^{40}$ We expect, nevertheless, that in an extended and generalized model, with endogenous innovation in the alternative energy sector, the fossil steady state can continue to coexist with the equilibrium without fossil. While the cost share of fossil resources might tend to rise because of knowledge-stock spillovers, it might also become increasingly costly in a growing economy to generate all energy from other sources than fossil: renewable energy technologies like wind and solar are subject to intermittency and scalability problems. Innovation in the alternative energy sector might offset these diminishing returns, which requires knowledge growth to be faster in this sector than in the non-energy sectors. But then production and innovation in fossil and non-fossil energy sectors become much more similar and investment decisions determine which of the two energy sources survives in the long run. The key insight from our paper is that complementarity of investment decisions of energy resource owners and energy users creates a multiplicity of equilibria which in principle generalizes to a setting with spillovers and many types of innovation. We leave it for future research to further investigate these extensions of our model.

\footnotetext{
${ }^{39}$ To model flow spillovers, we replace (6) by $\dot{q}_{R k}=\xi_{R} Q_{R}\left(D_{R k}+\zeta \hat{Q}_{L} D_{R}\right)$ and $\dot{q}_{L k}=\xi_{L} Q_{L} D_{L k}$, which implies that provided the R-sector as a whole undertakes research $\left(D_{R}>0\right)$, it receives spillovers from the other research sector. Integrating over firms $k$, we then find for relative knowledge growth: $\hat{Q}_{R}-\hat{Q}_{L}=$ $\xi_{R} D_{R}-\xi_{L} D_{L}+\zeta \xi_{R} \xi_{L} D_{L} D_{R}$. Hence, as before, a balanced growth path with knowledge stocks growing at different rates and constant labor allocation is feasible.

${ }^{40}$ To model stock spillovers consistent with Acemoglu (2002) and Hart (2012), we replace (6) by $\dot{q}_{i k}=$ $\xi_{i} \Psi\left(Q_{i}, Q_{j}\right) D_{i k}$, where $\Psi($.$) is a measure of the aggregate knowledge stock on which innovation builds (for$ simplicity symmetric across the two types of innovation). To ensure endogenous growth as in the main text, the function $\Psi($.$) needs to feature constant returns to scale. Integrating over firms k$ and dividing by the stock, we find the following expression for the growth rate of knowledge: $\hat{Q}_{i}=\xi_{i} \Psi\left(1, Q_{j} / Q_{i}\right) D_{i}$. It follows immediately that a balanced growth path with constant labor allocation ( $D_{i}$ constant) and constant growth rate of the relative knowledge stocks $\hat{Q}_{R}-\hat{Q}_{L}$ is only feasible if $Q_{L}$ and $Q_{R}$ grow at the same rate. However, a balanced growth path requires effective relative supply of production factors, $Q_{L} L / Q_{R} R$, to be constant, while $L / R$ grows. This in turn requires that the growth rate of relative technology $Q_{R} / Q_{L}$ can endogenously adjust and deviate from zero in the long run. Hence, we conclude that a balanced growth path does not arise with stock spillovers.
} 


\section{Appendix}

\section{A.1 Decentralized market equilibrium}

\section{A.1.1 Intermediate goods producers}

The current-value Hamiltonian associated with the optimization problem of firm $k$ in the intermediate good sector is given by:

$$
\mathcal{H}_{i k}=p_{Y i}(1-\beta) q_{i k} M_{i}^{\beta} x_{i k}^{1-\beta}-q_{i k} p_{Y} x_{i k}-w_{D} D_{i k}+\lambda_{i k} \xi_{i} Q_{i} D_{i k},
$$

where $i=M_{i}=\{R, L\}$. According to the Maximum Principle, the necessary first-order conditions for an optimum are given by:

$$
\begin{aligned}
& \frac{\partial \mathcal{H}_{i k}}{\partial x_{i k}}=0 \Rightarrow(1-\beta)^{2} p_{Y i} q_{i k} M_{i}^{\beta} x_{i k}^{-\beta}=q_{i k} p_{Y}, \\
& \frac{\partial \mathcal{H}_{i k}}{\partial D_{i k}} \leq 0 \Rightarrow-w_{D}+\lambda_{i k} \xi_{i} Q_{i} \leq 0, \quad \text { with equality if } \quad D_{i k}>0, \\
& \frac{\partial \mathcal{H}_{i k}}{\partial q_{i k}}=-\dot{\lambda}_{i k}+r \lambda_{i k} \Rightarrow p_{Y i}(1-\beta) M_{i}^{\beta} x_{i k}^{1-\beta}-x_{i k} p_{Y}=-\dot{\lambda}_{i k}+r \lambda_{i k} .
\end{aligned}
$$

The transversality conditions are given by (11a)-(11b). Substitution of (5b) in (A.2) gives (7a), (A.3) directly implies (7b), and the combination of (A.2) and (A.4) gives (7c).

\section{A.1.2 Households}

The current-value Hamiltonian associated with the households' optimization problem reads:

$$
\mathcal{H}^{M}=\ln C+\phi_{V}\left[r V+w_{R} R+w L^{S}-p_{Y} C\right]-\phi_{S} R
$$

According to the Maximum Principle, the necessary first-order conditions for an optimum are

$$
\begin{aligned}
& \frac{\partial \mathcal{H}^{M}}{\partial C}=0 \Rightarrow \frac{1}{C}-\phi_{V} p_{Y}=0 \Rightarrow \hat{C}+\hat{p}_{Y}=-\hat{\phi}_{V}, \\
& \frac{\partial \mathcal{H}^{M}}{\partial R}=0 \Rightarrow \phi_{V} w_{R}-\phi_{S}=0 \Rightarrow \hat{\phi}_{V}+\hat{w}_{R}=\hat{\phi}_{S}, \\
& \frac{\partial \mathcal{H}^{M}}{\partial S}=-\dot{\phi}_{S}+\rho \phi_{S} \Rightarrow \dot{\phi}_{S}+\rho \phi_{S}=0 \Rightarrow \hat{\phi}_{S}=\rho, \\
& \frac{\partial \mathcal{H}^{M}}{\partial V}=-\dot{\phi}_{V}+\rho \phi_{V} \Rightarrow \phi_{V} r=-\dot{\phi}_{V}+\rho \phi_{V} \Rightarrow \hat{\phi}_{V}=\rho-r .
\end{aligned}
$$

Combining (A.6a) and (A.6d) gives the Ramsey rule (14a). The first-order conditions (A.6b)(A.6d) yield the Hotelling rule (14b). 


\section{A.1.3 Proof of Lemma 1}

Condition (7b) holds with equality in both intermediate goods sectors if $D_{L}>0$ and $D_{R}>0$. Therefore, the return to quality improvements in both sectors is described by (9). Aggregating (6) over all firms in the sector, we find $\hat{Q}_{i}=\xi_{i} D_{i}$. Combining this expression with (9), we get an equation that relates the rate of return in the fossil economy to the aggregate research level:

$$
r-\hat{w}_{L}=\frac{L^{S} \beta(1-\beta)}{\mu_{L}}-D_{L}-D_{R},
$$

Substituting (A.7) into (9), we find that the endogenous bias in the direction of technical change is given by

$$
\hat{Q}_{R}-\hat{Q}_{L}=\frac{L^{S} \beta(1-\beta)}{\mu_{L}} \xi_{L} \xi_{R}\left(\theta_{R}-\xi_{R}^{-1}\right) .
$$

Equation (A.8) shows that the bias in technical progress depends on the resource income share: if the resource is scarce and therefore the resource income share is large, technical change will be relatively resource-augmenting and vice versa.

Differentiating $\mu_{L}$ with respect to time, using the Ramsey rule (14a) and substituting (A.7), we obtain

$$
\dot{\mu}_{L}=\mu_{L}\left(D_{L}+D_{R}+\rho\right)-L^{S} \beta(1-\beta) .
$$

Combining (A.7)-(A.9) with (18) and (20) gives (22a)-(22b).

\section{A.1.4 Proof of Lemma 2}

By using (6), (9), and (14a), and imposing $D_{R}=0$ in (9) and (20), we obtain (24a)-(24b).

\section{A.1.5 Proof of Lemma 3}

Energy generation with the backstop technology takes place according to (3b). Perfect competition implies that the price of energy generated with the backstop technology is equal to its marginal production cost: $p_{Y H}=p_{Y} / \eta$. Using this equality in $p_{Y H}=p_{Y} \partial Y / \partial Y_{H}$ together with the factor demand equations (2a)-(2b), we obtain (25a). Because the backstop economy is characterized by purely labor-augmenting technical progress, (24a) is still valid. Combining (24a) and (25a) we obtain the steady-state full labor income share (25b). 


\section{A.1.6 Ratios and growth rates in terms of income shares}

Several observable macroeconomic variables can be easily obtained from the path through the $\left(\mu_{L}, \theta_{R}\right)$-plane. We first derive expressions for factor, price and output ratios, and subsequently for the growth rate of output. The fraction of labor devoted to production follows from using the definition of $\mu_{L}$ in (18): $L / L^{S}=\beta\left(1-\theta_{E}\right) / \mu_{L}$. Furthermore, the combination of (1), and (2a)-(2b) gives the following expressions for relative prices and sectoral output shares

$$
\begin{aligned}
\frac{p_{Y L}}{p_{Y}} & =\left(\frac{1-\theta_{E}}{\gamma^{\sigma}}\right)^{\frac{1}{1-\sigma}}, \quad \frac{p_{Y E}}{p_{Y}}=\left(\frac{\theta_{E}}{(1-\gamma)^{\sigma}}\right)^{\frac{1}{1-\sigma}}, \\
\frac{Y_{L}}{Y} & =\left(\frac{1-\theta_{E}}{\gamma}\right)^{\frac{\sigma}{1-\sigma}}, \quad \frac{Y_{E}}{Y}=\left(\frac{\theta_{E}}{1-\gamma}\right)^{\frac{\sigma}{1-\sigma}} .
\end{aligned}
$$

To find an expression for the growth rate of output, we start by combining (1), (2a)-(2b), (4), (8), and (16) to obtain the level of output:

$$
\left.Y=\frac{Q_{L}}{\mu_{L}} L^{S} \gamma^{\frac{\sigma}{\nu-1}} \beta(1-\beta)^{2 \frac{1-\beta}{\beta}}\left(1-\theta_{E}\right)\right)^{\frac{1}{1-\nu}}
$$

By taking the growth rate of this expression and by substituting (7b)-(7c) and (8) into the result, we find

$$
\hat{Y}=\beta(1-\beta) \frac{1-\theta_{R}}{\mu_{L}} \xi_{L} L^{S}-\frac{1}{\left(1-\theta_{E}\right)(1-\nu)} \dot{\theta}_{E}-\rho
$$

Substitution of (22a) or (24a) into (A.13) gives an expression for output growth in terms of $\theta_{R}$ and $\mu_{L}$ in the resource regime with both types of technical progress or with only laboraugmenting technical progress, respectively. In the steady state of the resource regime, and throughout the backstop regime, $\dot{\theta}_{E}=0$. Substitution of this equality together with (23a)(23b) and (25b) into (A.13) gives closed-form expressions for the growth rates of output in the steady state of the resource regime and in the backstop regime, respectively:

$$
\hat{Y}^{*}=\frac{1-\beta}{1+\xi_{L}(1-\beta)}\left(\xi_{L} L^{S}+\rho\right)-\rho, \quad \hat{Y}^{H}=\frac{1-\beta}{2-\beta}\left(\xi_{L} L^{S}+\rho\right)-\rho,
$$

where an asterisk again denotes the steady state, and the subscript $H$ indicates the backstop regime.

\section{A.1.7 Extraction isoclines}

We substitute (22b) or (24b) into (21) to find the following expressions for the extraction isoclines in the fossil economy with both types of technical progress or with only labor-augmenting 
technical progress, respectively:

$$
\begin{aligned}
\left.\mu_{L}\right|_{\dot{R}=0, D_{L}>0, D_{R}>0} & =\frac{\beta L^{S}\left(1-\theta_{R}\right)(1-\nu)}{\rho+L^{S}\left(1-\theta_{R}\right)(1-\nu)}\left[1-\theta_{R}+(1-\beta)\left(1+\xi_{L}-\xi_{L} \xi_{R} \theta_{R}\right)\right], \\
\left.\mu_{L}\right|_{\dot{R}=0, D_{L}>0, D_{R}=0} & =\frac{\xi_{L} L^{S} \beta(1-\beta)(1-\nu)}{\rho}\left(1-\theta_{R}\right)^{2} .
\end{aligned}
$$

\section{A.1.8 Simultaneous energy use regime}

We show that it is not possible to have a regime of simultaneous use of the resource and the backstop technology. Simultaneous use requires equal effective prices of the resource and the backstop technology, so that $p_{Y H}=p_{Y R}=p_{Y E}$. Using $p_{Y H}=p_{Y} \partial Y / \partial Y_{H}=p_{Y} / \eta$, this implies $\theta_{E}=(1-\gamma)^{\sigma} \eta^{\sigma-1}$ and

$$
\hat{p}_{Y}=\hat{p}_{Y H}=\hat{p}_{Y R}=\hat{p}_{Y E} .
$$

If we combine (4) with (5a), and (5b) to find the prices $p_{Y L}$ and $p_{Y R}$, and subsequently convert the expressions into growth rates, we get

$$
\begin{aligned}
& \hat{p}_{Y L}-\hat{p}_{Y}=\beta\left(\hat{w}_{L}-\hat{p}_{Y}-\hat{Q}_{L}\right), \\
& \hat{p}_{Y R}-\hat{p}_{Y}=\beta\left(\hat{w}_{R}-\hat{p}_{Y}-\hat{Q}_{R}\right) .
\end{aligned}
$$

Using (2a)-(2b) together with (A.15) and the constancy of $\theta_{E}$, we find $\hat{p}_{Y L}=\hat{p}_{Y}$. Substitution of this result into (A.16a) and (A.15) into (A.16b), and using the Hotelling rule (14b), we obtain $r-\hat{w}_{D}=\hat{Q}_{R}-\hat{Q}_{L}$. Substitution of (18) into (9), in a regime with purely laboraugmenting technical change (i.e., $\hat{Q}_{L}>0$ and $\left.\hat{Q}_{R}=0\right)$ we have $r-\hat{w}_{D}=(1-\beta) \xi_{L}\left(L^{S}-\right.$ $D)-\hat{Q}_{L}$. The latter two conditions can only be satisfied jointly if $D=L^{S}$. However, this implies that $L=Y=0$, which cannot hold in equilibrium because it implies $\hat{C}=\hat{Y}=0$, whereas the Ramsey rule (14a) together with (A.16b) gives $\hat{C}=-\rho$. Hence, during a regime with purely labor-augmenting technical change, the effective relative price of the resource and the backstop cannot be constant, so that simultaneous use of both energy sources will not occur. As a result, simultaneous use is also impossible in a regime with both resource-augmenting and labor-augmenting technical change. Let us proof this by contradiction: assume that a regime of simultaneous use with both resource-augmenting and labor-augmenting technical change exists. During this regime, we must have $\theta_{E}=(1-\gamma)^{\sigma} \eta^{\sigma-1}$ due to equal prices of the resource and the backstop. Optimality condition (7b) together with (A.17) implies that the economy before the switch to the backstop necessarily shifts to a regime with purely labor-augmenting technical change, during which $\dot{\theta}_{E}>0$, according to (24b), which implies that $\theta_{E}<(1-$ 
$\gamma)^{\sigma} \eta^{\sigma-1}$ during the preceding simultaneous use regime with both resource-augmenting and labor-augmenting technical change. Hence, we get a contradiction.

\section{A.1.9 Proof of Lemma 5}

We solve the differential equation (7c), using the transversality condition (11b), and find the solution for the shadow price at time $T$ :

$$
\lambda_{R}(T)=\int_{T}^{\infty} \frac{\beta}{1-\beta} x_{R}(t) p_{Y}(t) e^{\int_{T}^{t} r(s) d s} d t
$$

At $T$, we have $\lambda_{R}(T)=0$ because $x_{R}(t)=0$ for $t>T$. Hence, the shadow price of quality in the resource service sector equals zero at the moment the economy switches from the resource to the backstop. Continuity of the shadow price then implies that just before the switch, (7b) for the resource service sector holds with inequality, yielding $D_{R}=0$. This proves part (i). ${ }^{41}$

As argued in the main text, continuity of consumption at $t=T$ requires $Y\left(T^{-}\right)=(1-$ $\left.\theta_{H}^{*}\right) Y\left(T^{+}\right) .{ }^{42}$ Because prices are continuous in a market equilibrium, (2a)-(2b) imply that income shares are continuous as well. Hence, by using (A.12) we find that the jump in $\mu_{L}$ must be inversely proportional to the jump in $Y$, which implies the result in part (ii).

\section{A.1.10 Proof of Lemma 6}

An internal solution with both types of technical progress occurring at a particular moment in time requires (7b) to hold with equality. From $\chi \equiv \xi_{R} Q_{R} \lambda_{R} / w_{L}$, we see that (7b) implies that resource-augmenting technical change is positive (zero) if $\chi=(<) 1$. To characterize the dynamics, we take the time derivative of $\chi$ and substitute (7c), (9) and (18) to obtain a differential equation that describes the evolution of $\chi$ during the purely labor-augmenting $R \& D$ regime:

$$
\dot{\chi}=\left[\frac{\beta(2-\beta) L^{S}}{\mu_{L}} \xi_{L}\left(1-\theta_{R}\right)-\xi_{L} L^{S}\right] \chi-\frac{\beta(1-\beta) L^{S}}{\mu_{L}} \xi_{R} \theta_{R} .
$$

\footnotetext{
${ }^{41}$ This result of the existence of a regime without resource-augmenting technical change before the switch to the backstop, does not rely on our assumption of sector-specific learning, i.e., absence of intersectoral spillovers, which is implicit in (6) (cf. footnote 10). The same result holds under 'limited state dependence', i.e., when the productivity of researchers does not only depend on knowledge in their own sector, but also on accumulated knowledge in the other sector, as in Acemoglu (2002). However, in that case our model would feature 'semi-endogenous' growth from the start of the $D_{R}=0$ regime onwards and without population growth output growth would be zero in the long run (cf. Jones, 1995).

${ }^{42}$ Continuity of consumption follows from the Ramsey rule (14a) and the finiteness of the rate of interest from (6), (7b), (9), and the continuity of the shadow price $\lambda_{L k}$. Equivalently, continuity of consumption follows from the continuity of the co-state variable $\phi_{S}$ (due to (A.6c)) in the Hamiltonian (A.5), when we use $Y$ as the numéraire, implying $p_{Y}=1$.
} 
Entering (leaving) the simultaneous-R\&D regime requires $\dot{\chi} \geq(<) 0$ at $\chi=1$, which from (A.18) requires $\mu_{L} \leq(>) \beta(2-\beta)-\beta\left[1+(1-\beta) \xi_{R}\right] \theta_{R}$ implying that $D_{R} \leq(>) 0$ from the second condition in footnote 18 . Since we know from the second condition in footnote 18 that the simultaneous- $R \& D$ regime is impossible if $\mu_{L}<\beta(2-\beta)-\beta\left[1+(1-\beta) \xi_{R}\right] \theta_{R}$, the economy can only enter the simultaneous- $R \& D$ regime if $\mu_{L}=\beta(2-\beta)-\beta\left[1+(1-\beta) \xi_{R}\right] \theta_{R}$.

\section{A.2 Social optimum}

The social planner solves the following maximization problem:

$$
\max \int_{0}^{\infty} \ln C(t) e^{-\rho t} d t
$$

subject to (1), (3a), (3b), (4), (12), (6), (17), $D_{L k} \geq 0, D_{R k} \geq 0, Y_{R} \geq 0, Y_{H} \geq 0$, with $k \in[0,1]$, by choosing time paths for the control variables $C, D_{L k}, D_{R k}, L, R, x_{L k}, x_{R k}, Y, Y_{L}$, $Y_{E}$, and $Y_{R}$. The state variables are $q_{L k}, q_{R k}$, and $S$. The associated current-value Hamiltonian and Lagrangian read, respectively: ${ }^{43}$

$$
\mathcal{H}^{S}=\ln C+\int_{0}^{1} \lambda_{q L k} \xi_{L} Q_{L} D_{L k} d k+\int_{0}^{1} \lambda_{q R k} \xi_{R} Q_{R} D_{R k} d k-\lambda_{S} R,
$$

$$
\begin{aligned}
\mathcal{L}= & \mathcal{H}-\mu_{C}\left(C-Y+\int_{0}^{1} q_{L k} x_{L k} d k+\int_{0}^{1} q_{R k} x_{R k} d k+\frac{1}{\eta} Y_{H}\right) \\
& -\mu_{Y}\left[Y-\left(\gamma Y_{L}^{\frac{\sigma-1}{\sigma}}+(1-\gamma) Y_{E}^{\frac{\sigma-1}{\sigma}}\right)^{\frac{\sigma}{\sigma-1}}\right]-\mu_{Y E}\left(Y_{E}-Y_{R}-Y_{H}\right) \\
& -\mu_{Y L}\left(Y_{L}-L^{\beta} \int_{0}^{1} q_{L k} x_{L k}^{1-\beta} d k\right)-\mu_{Y R}\left(Y_{R}-R^{\beta} \int_{0}^{1} q_{R k} x_{R k}^{1-\beta} d k\right) \\
& -\mu_{L M E}\left(D_{L}+D_{R}+L-L^{S}\right)-\int_{0}^{1} \zeta_{D L k} D_{L k}-\int_{0}^{1} \zeta_{D R k} D_{R k}-\zeta_{Y H} Y_{H}-\zeta_{Y R} Y_{R} .
\end{aligned}
$$

\footnotetext{
${ }^{43}$ Note that, to facilitate the comparison between (7a)-(7c) and (28a)-(28c), in Section 5.1 we have used $p$ and $w$ instead of $\mu$ for shadow prices, and we have defined $p_{x i k}$ by using (5b).
} 
The first-order conditions are given by
(A.21a) $\quad \frac{\partial \mathcal{L}}{\partial C}=\frac{1}{C}-\mu_{C}=0$,
(A.21b) $\quad \frac{\partial \mathcal{L}}{\partial Y}=\mu_{C}-\mu_{Y}=0$,
(A.21c) $\frac{\partial \mathcal{L}}{\partial Y_{L}}=\mu_{Y} \gamma Y_{L}^{-\frac{1}{\sigma}}\left(\gamma Y_{L}^{\frac{\sigma-1}{\sigma}}+(1-\gamma) Y_{E}^{\frac{\sigma-1}{\sigma}}\right)^{\frac{1}{\sigma-1}}-\mu_{Y L}=0$,
(A.21d) $\quad \frac{\partial \mathcal{L}}{\partial Y_{E}}=\mu_{Y}(1-\gamma) Y_{E}^{-\frac{1}{\sigma}}\left(\gamma Y_{L}^{\frac{\sigma-1}{\sigma}}+(1-\gamma) Y_{E}^{\frac{\sigma-1}{\sigma}}\right)^{\frac{1}{\sigma-1}}-\mu_{Y E}=0$,
(A.21e) $\quad \frac{\partial \mathcal{L}}{\partial Y_{H}}=\mu_{Y E}-\frac{\mu_{C}}{\eta}-\zeta_{Y H}=0$,
(A.21f) $\quad \frac{\partial \mathcal{L}}{\partial Y_{R}}=\mu_{Y E}-\mu_{Y R}-\zeta_{Y R}=0$,
(A.21g) $\frac{\partial \mathcal{L}}{\partial D_{L k}}=\lambda_{q L k} \xi_{L} Q_{L}-\mu_{L M E}-\zeta_{L k}=0, \forall k \in[0,1]$,
(A.21h) $\frac{\partial \mathcal{L}}{\partial D_{R k}}=\lambda_{q R k} \xi_{R} Q_{R}-\mu_{L M E}-\zeta_{R k}=0, \forall k \in[0,1]$,
(A.21i) $\frac{\partial \mathcal{L}}{\partial x_{L k}}=-\mu_{C} q_{L k}+\mu_{Y L}(1-\beta) L^{\beta} q_{L k} x_{L k}^{-\beta}=0, \quad \forall k \in[0,1]$,
(A.21j) $\quad \frac{\partial \mathcal{L}}{\partial x_{R k}}=-\mu_{C} q_{R k}+\mu_{Y R}(1-\beta) R^{\beta} q_{R k} x_{R k}^{-\beta}=0, \quad \forall k \in[0,1]$,
(A.21k) $\quad \frac{\partial \mathcal{L}}{\partial L}=\mu_{Y L} \beta L^{\beta-1} \int_{0}^{1} q_{L k} x_{L k}^{1-\beta} d k-\mu_{L M E}=0$,
(A.211) $\quad \frac{\partial \mathcal{L}}{\partial R}=\mu_{Y R} \beta R^{\beta-1} \int_{0}^{1} q_{R k} x_{R k}^{1-\beta} d k-\lambda_{S}=0$,
(A.21m) $\quad \frac{\partial \mathcal{L}}{\partial q_{L k}}=\lambda_{q L k} \xi_{L} D_{L k}-\mu_{C} x_{L k}+\mu_{Y L} L^{\beta} x_{L k}^{1-\beta}=-\dot{\lambda}_{q L k}+\rho \lambda_{q L k}, \quad \forall k \in[0,1]$,
(A.21n) $\frac{\partial \mathcal{L}}{\partial q_{R k}}=\lambda_{q R k} \xi_{R} D_{R k}-\mu_{C} x_{R k}+\mu_{Y R} R^{\beta} x_{R k}^{1-\beta}=-\dot{\lambda}_{q R k}+\rho \lambda_{q R k}, \quad \forall k \in[0,1]$,
(A.21o) $\quad \frac{\partial \mathcal{L}}{\partial S}=0=-\dot{\lambda}_{S}+\rho \lambda_{S}$.

The Kuhn-Tucker conditions are

(A.22)

$$
\zeta_{D L k} D_{L k}=0, \quad \zeta_{D R k} D_{R k}=0, \quad \zeta_{Y H} Y_{H}=0, \quad \zeta_{Y R} Y_{R}=0
$$

The transversality conditions read

$$
\lim _{t \rightarrow \infty} \lambda_{q L k} q_{L k} e^{-\rho t}=0, \quad \lim _{t \rightarrow \infty} \lambda_{q R k} q_{R k} e^{-\rho t}=0, \quad \lim _{t \rightarrow \infty} \lambda_{S} S=0
$$

From (A.21i)-(A.21j) we get $x_{L k}=x_{L}$ and $x_{R k}=x_{R}$ for all $k \in[0,1]$. Furthermore, (A.21g), (A.21m), and (A.23) ((A.21h), (A.21n), and (A.23)) yield $\lambda_{q L k}=\lambda_{q L}, \zeta_{L k}=\zeta_{L}$, and $D_{L k}=D_{L}$ 
$\left(\lambda_{q R k}=\lambda_{q R}, \zeta_{R k}=\zeta_{R}\right.$ and $\left.D_{R k}=D_{R}\right)$ for all $k \in[0,1]$. For the output elasticities we have

$$
\theta_{L} \equiv \frac{\left(\partial Y / \partial Y_{L}\right) Y_{L}}{Y}=\frac{\mu_{Y L} Y_{L}}{\mu_{Y} Y}, \quad \theta_{E} \equiv \frac{\left(\partial Y / \partial Y_{E}\right) Y_{E}}{Y} \frac{\mu_{Y E} Y_{E}}{\mu_{Y} Y}
$$

where the equalities use (A.21c)-(A.21d). The first-order conditions (A.21a)-(A.21o) can be condensed to the following dynamical system:

$$
\begin{aligned}
\mu_{Y L} \frac{\partial Y_{L}}{\partial L} & =\mu_{L M E} \Rightarrow \beta Y \mu_{Y} \frac{\theta_{L}}{L}=\mu_{L M E} \\
\mu_{Y R} \frac{\partial Y_{R}}{\partial R} & =\lambda_{S} \Rightarrow \beta Y \mu_{Y} \frac{\theta_{R}}{R}=\lambda_{S}, \\
\mu_{L M E} & =\lambda_{q L} \xi_{L} Q_{L}-\zeta_{L}, \\
\mu_{L M E} & =\lambda_{q R} \xi_{R} Q_{R}-\zeta_{R}, \\
\xi_{L} D_{L}+\beta Y \mu_{Y} \frac{\theta_{L}}{\lambda_{q L} Q_{L}} & =-\hat{\lambda}_{q L}+\rho \\
\xi_{R} D_{R}+\beta Y \mu_{Y} \frac{\theta_{R}}{\lambda_{q R} Q_{R}} & =-\hat{\lambda}_{q R}+\rho \\
\hat{\lambda}_{S} & =\rho
\end{aligned}
$$

where (A.25a)-(A.25b) are obtained by rewriting (A.21k) and (A.211); (A.25c)-(A.25d) follow from (A.21g)-(A.21h); (A.25e)-(A.25f) can be found by substituting (A.21c) and (A.21i) into (A.21m), and (A.21d) and (A.21j) into (A.21n); and (A.25g) is immediate from (A.21o).

Depending on the values of the Lagrange multipliers $\zeta_{Y H}$ and $\zeta_{Y R}$ there are different regimes of energy use. Within each energy regime, there also potentially exist different technology regimes, depending on the values of $\zeta_{D L}$ and $\zeta_{D R}$. The different regimes will be discussed in turn.

\section{A.2.1 Resource regime}

In the resource regime, $\zeta_{Y H}>0$ and $\zeta_{Y R}=0$, which implies $Y_{H}=0$ and $Y_{R}>0$. It follows from these implications and from (A.21e) that

$$
\mu_{Y E}=\frac{\mu_{C}}{\eta}+\zeta_{Y H} \Rightarrow \mu_{Y E}<\frac{\mu_{C}}{\eta} .
$$

Combining this inequality with (A.21b) and (A.21d) we find that during the resource regime we have

$$
\frac{\mu_{Y}}{\eta}>\mu_{Y}(1-\gamma)\left(\frac{Y}{Y_{E}}\right)^{\frac{1}{\sigma}} \Rightarrow \theta_{E}=\theta_{R}<\theta_{H}^{*} \equiv(1-\gamma)^{\sigma} \eta^{\sigma-1}
$$


Furthermore, we obtain from (A.21b), (A.21i)-(A.21j), and (4)

$$
x_{L}=\frac{(1-\beta) \theta_{L} Y}{Q_{L}}, \quad x_{R}=\frac{(1-\beta) \theta_{R} Y}{Q_{R}} .
$$

Using (A.28) in (15) we find

$$
C=\beta Y \text {. }
$$

Finally, we combine (A.21c)-(A.21f) and (A.21i)-(A.211) to find

$$
\frac{\theta_{L}}{\theta_{R}}=\left(\frac{\gamma}{1-\gamma}\right)^{\sigma}\left(\frac{Q_{R} / \lambda_{S}}{Q_{L} / \mu_{L M E}}\right)^{1-\nu}
$$

Internal solution: both types of technical progress In an internal solution with $\zeta_{L}=\zeta_{R}=0$, (6) and (A.25c)-(A.25d) imply

$$
\lambda_{q L} \xi_{L} Q_{L}=\lambda_{q R} \xi_{R} Q_{R} \Rightarrow \hat{\lambda}_{q L}+\hat{Q}_{L}=\hat{\lambda}_{q R}+\hat{Q}_{R}
$$

Using this result in (A.25e)-(A.25f) gives

$$
\frac{1-\theta_{R}}{\lambda_{q L} Q_{L}}=\frac{\theta_{R}}{\lambda_{q R} Q_{R}} \Rightarrow \frac{\theta_{R}}{1-\theta_{R}}=\frac{\xi_{R}^{-1}}{\xi_{L}^{-1}} .
$$

We use the normalization $\xi_{L}^{-1}+\xi_{R}^{-1}=1$ to rewrite (A.32) as $\theta_{R}=\xi_{R}^{-1}$. From (A.29), (A.25c) and (A.25e) we get

$$
\hat{\mu}_{L M E}=\hat{\lambda}_{q L}+\hat{Q}_{L}=\rho-\frac{\theta_{L}}{\lambda_{q L} Q_{L}}=\rho-\frac{\xi_{L}}{\mu_{L M E}}\left(1-\theta_{R}\right) .
$$

Substitution of (A.32) gives

$$
\dot{\mu}_{L M E}=\rho \mu_{L M E}-1
$$

which is an unstable differential equation in $\mu_{L M E}$. Hence (A.32)-(A.34) together imply that an internal solution with $D_{L}>0$ and $D_{R}>0$ only occurs in the steady state of the model, with constant $\theta_{R}=\xi_{R}^{-1}$ and $\mu_{L M E}=\rho^{-1}$. Using these expressions for $\theta_{R}$ and $\mu_{L M E}$ and the combination of (1), (4), (6), (17), (A.21c)-(A.21d), (A.21f), (A.25a), (A.25g), (A.28), and (A.30) we also find $\hat{Y}=\hat{Q}_{L}=L^{S}-\rho$ in the steady state. The transition to this steady state is characterized by corner solutions with either $D_{L}=0$ or $D_{R}=0$. 
Corner solutions: one type of technical progress We define the following ratio of marginal products of $R \& D$

$$
z \equiv \frac{\lambda_{q R} \xi_{R} Q_{R}}{\lambda_{q L} \xi_{L} Q_{L}}
$$

It follows from (A.25c)-(A.25d) that $D_{L}=0\left(D_{R}=0\right)$ if $z>1(z<1)$. We rewrite (A.30) in terms of growth rates to get

$$
\hat{\theta}_{R}=\left(1-\theta_{R}\right)(1-\nu)\left[\hat{Q}_{L}-\hat{Q}_{R}+\hat{\lambda}_{S}-\hat{\mu}_{L M E}\right]
$$

The canonical systems that characterize the two corner solutions are described in the following two lemmata.

Lemma A.1 If $z<1$, then $D_{L}>0, D_{R}=0$ and the dynamical system is described by

$$
\begin{aligned}
\dot{\theta}_{R} & =\theta_{R}\left(1-\theta_{R}\right)(1-\nu) \xi_{L} L^{S}, \\
\dot{\mu}_{L M E} & =\rho \mu_{L M E}-\left(1-\theta_{R}\right) \xi_{L}, \\
\dot{z} & =\frac{\xi_{R}}{\mu_{L M E}}\left[\left(1-\theta_{R}\right) \frac{\xi_{L}}{\xi_{R}} z-\theta_{R}\right] .
\end{aligned}
$$

Proof. First, it follows from (A.25c)-(A.25d) that $D_{L}>0$ and $D_{R}=0$ if $z<1$. Second, by using (A.25a), (A.25c) and (A.25e) we obtain $\hat{\mu}_{L M E}=\rho-\xi_{L} L$. Substitution of this result together with $\hat{Q}_{R}=0$ into (A.36) gives (A.37a). Subsequently, we combine (A.29), (A.25c) and (A.25e) to obtain

$$
\hat{\mu}_{L M E}=\hat{\lambda}_{q L}+\hat{Q}_{L}=\rho-\frac{\theta_{L}}{\lambda_{q L} Q_{L}}=\rho-\frac{\xi_{L}}{\mu_{L M E}}\left(1-\theta_{R}\right),
$$

which gives (A.37b) upon multiplying by $\mu_{L M E}$. Finally, rewrite (A.35) into growth rates to get

$$
\hat{z}=\hat{\lambda}_{q R}+\hat{Q}_{R}-\left(\hat{\lambda}_{q L}+\hat{Q}_{L}\right)
$$

Substitution of (A.29), (A.25c), and (A.25e)-(A.25f) gives

(A.40) $\dot{z}=\frac{\theta_{L}}{\lambda_{q L} Q_{L}} z-\frac{\xi_{R}}{\xi_{L}} \frac{\theta_{R}}{\lambda_{q L} Q_{L}}=\xi_{L} L z-\xi_{R} L \frac{\theta_{R}}{1-\theta_{R}}=\frac{\xi_{R}}{\mu_{L M E}}\left[\left(1-\theta_{R}\right) \frac{\xi_{L}}{\xi_{R}} z-\theta_{R}\right]$. 
Lemma A.2 If $z>1$, then $D_{R}>0, D_{L}=0$ and the dynamical system is described by

$$
\dot{\theta}_{R}=\theta_{R}\left(1-\theta_{R}\right)(1-\nu) \xi_{R} \frac{1-L^{S} \mu_{L M E}}{\mu_{L M E}},
$$

$$
\dot{\mu}_{L M E}=\rho \mu_{L M E}-\theta_{R} \xi_{R}
$$

$$
\dot{z}=\frac{\xi_{R}}{\mu_{L M E}} z\left[\left(1-\theta_{R}\right) \frac{\xi_{L}}{\xi_{R}} z-\theta_{R}\right] .
$$

Proof. First, it follows from (A.25c)-(A.25d) that $D_{L}=0$ and $D_{R}>0$ if $z>1$. Second, by using (A.25a), (A.25d) and (A.25f) we obtain $\hat{\mu}_{L M E}=\rho-\theta_{R} \xi_{R} \frac{L}{\theta_{L}}$. Substitution of this result together with $\hat{Q}_{L}=0$ into (A.36) gives (A.41a). Subsequently, we combine (A.29), (A.25d) and (A.25f) to obtain

$$
\hat{\mu}_{L M E}=\hat{\lambda}_{q R}+\hat{Q}_{R}=\rho-\frac{\theta_{R}}{\lambda_{q R} Q_{R}}=\rho-\frac{\xi_{R}}{\mu_{L M E}} \theta_{R}
$$

which gives (A.41b) upon multiplying by $\mu_{L M E}$. Finally, substitute (A.29), (A.25d), and (A.25e)(A.25f) into (A.39) to get (A.41c).

\section{A.2.2 Backstop regime}

In the backstop regime, $\zeta_{Y H}=0$ and $\zeta_{Y R}>0$, which implies $Y_{H}>0$ and $Y_{R}=0$. It follows from these implications and from (A.21e) that $\mu_{Y E}=\mu_{C} / \eta$. Combining this equality with (A.21b) and (A.21d) we find that during the resource regime we have

$$
\frac{\mu_{Y}}{\eta}=\mu_{Y}(1-\gamma)\left(\frac{Y}{Y_{E}}\right)^{\frac{1}{\sigma}} \Rightarrow \theta_{E}=\theta_{H}^{*}=(1-\gamma)^{\sigma} \eta^{\sigma-1} .
$$

If the backstop regime starts at $T_{H}$ and continuous forever, first-order condition (A.21o) and the third transversality condition in (A.23) imply $S\left(T_{H}\right)=0$, because

$$
\lim _{t \rightarrow \infty} \lambda_{S}(t) S\left(T_{H}\right) e^{-\rho t}=\lim _{t \rightarrow \infty} \lambda_{S}\left(T_{H}\right) S\left(T_{H}\right) .
$$

Furthermore, solving the differential equation (A.25f) we find

$$
\lambda_{q R}\left(T_{H}\right)=\int_{T_{H}}^{\infty} \frac{\theta_{R}(t)}{1-\theta_{R}(t)} \frac{1}{Q_{R}(t)} e^{-\rho\left(t-T_{H}\right)} d t=0,
$$

where we have used $D_{R}=0$, because if $D_{R}$ would be positive, $\hat{\mu}_{L M E}=0$ and (A.25d) would imply

$$
\hat{\lambda}_{q R}+\hat{Q}_{R}=\rho-\frac{\theta_{R}}{1-\theta_{R}} \frac{1}{\lambda_{q R} Q_{R}}=0,
$$


which is impossible given that $\theta_{R}=0$ because $S=R=0$ in the backstop regime. ${ }^{44}$ Finally, we get from (A.21e), and (A.24) that $\theta_{E}=\frac{1}{\eta} \frac{Y_{H}}{Y}$. Combining this with (15) and (A.28), we find

$$
C=\beta \theta_{L} Y
$$

Combine (A.25a), (A.25c), (A.25e), and (A.47) to get $\dot{\mu}_{L}=\rho \mu_{L}-\xi_{L}$, which is an unstable differential equation in $\mu_{L}$, implying that $\mu_{L}=\xi_{L} / \rho$. Using this expression together with the constancy of $\theta_{E}$ and combining (1), (4), (6), (17), (A.21c)-(A.21d), (A.21f), (A.25a), (A.28), and (A.47), we find $\hat{Y}=\hat{Q}_{L}=\xi_{L} L^{S}-\rho$ during the backstop regime.

\section{A.2.3 Simultaneous energy use regime}

Here we show that the resource and the backstop will not be simultaneously used in the optimum.

Lemma A.3 A regime of simultaneous use of the resource and the backstop does not exist.

Proof. First, note that in a regime with simultaneous use we have

$$
\mu_{Y E}=\mu_{Y R}=\mu_{Y H}=\frac{\mu_{C}}{\eta} \Rightarrow \hat{\mu}_{Y E}=\hat{\mu}_{Y R}=\hat{\mu}_{Y H}=\hat{\mu}_{C}=\hat{\mu}_{Y} .
$$

Combining the former equalities with with (A.21b) and (A.21d) we find that during a regime of simultaneous use we have

$$
\frac{\mu_{Y}}{\eta}=\mu_{Y}(1-\gamma)\left(\frac{Y}{Y_{E}}\right)^{\frac{1}{\sigma}} \Rightarrow \theta_{E}=(1-\gamma)^{\sigma} \eta^{\sigma-1} .
$$

Substitution of (A.48)-(A.49) in the combination of (A.21c)-(A.21d), we get $\hat{\mu}_{Y L}=\hat{\mu}_{Y E}$. Subsequently, we take the growth rates of expressions (A.21i)-(A.21j) to find $\hat{x}_{L}=\hat{L}$, and $\hat{x}_{R}=\hat{R}$. Furthermore, converting (A.21k)-(A.211) into growth rates, we obtain

$$
\begin{array}{r}
\hat{\mu}_{Y L}+(1-\beta)\left(\hat{x}_{L}-\hat{L}\right)+\hat{Q}_{L}=\hat{\mu}_{L M E}, \\
\hat{\mu}_{Y R}+(1-\beta)\left(\hat{x}_{R}-\hat{R}\right)+\hat{Q}_{R}=\hat{\lambda}_{S} .
\end{array}
$$

Combining these latter expressions, while using (A.21o) and imposing $\hat{Q}_{R}=\xi_{R} D_{R}=0$, we get

$$
\hat{Q}_{L}=\hat{\mu}_{L M E}-\rho .
$$

\footnotetext{
${ }^{44}$ Note that the equality $\lambda_{q R}\left(T_{H}\right)=0$ (and therefore the necessary existence of the fossil regime with $D_{R}=0$ immediately before the backstop regime) does not require the 'extreme state dependence' assumption in (6). More flexible formulations with 'limited state dependence' like $\dot{q_{L}}=\xi_{L} Q_{L}^{(1+\delta) / 2} Q_{R}^{(1-\delta) / 2} D_{L}$ and $\dot{q}_{R}=$ $\xi_{R} Q_{R}^{(1+\delta) / 2} Q_{L}^{(1-\delta) / 2} D_{R}$, with $\delta \in[0,1]$ (cf. Acemoglu, 2002) give the same result.
} 
Writing (A.25c) in terms of growth rates and using (A.51) yields $\hat{\lambda}_{q L}=\rho$. Substituting (A.25a) and (A.25c) into (A.25e) gives $\hat{Q}_{L}+\xi_{L} L=-\hat{\lambda}_{q L}+\rho$. Hence, we get $\hat{Q}_{L}=-\xi_{L} L<0$, which is impossible because $\hat{Q}_{L} \geq 0$. As a result, simultaneous use cannot occur in a regime with $D_{R}=0$. It follows from (A.25d), (A.45), and the continuity of the Lagrange multiplier $\lambda_{q R}$ that $\zeta_{R}>0$ (and thus $D_{R}=0$ ) during a non-degenerate interval of time at the end of the simultaneous use regime. Hence, simultaneous use cannot occur at all, because at the beginning of the final pre-backstop regime with $D_{R}=0, \theta_{E}<(1-\gamma)^{\sigma} \eta^{\sigma-1}$, according to (A.37a).

\section{A.2.4 Transition}

Given that simultaneous use of the resource and the backstop can be excluded, and given that resource extraction is costless, the optimum starts with $\zeta_{Y H}>0$, and $Y_{H}=0$. Furthermore, if the optimum does not start in the steady state with $\theta_{E}=\xi_{R}^{-1}$ and $\mu_{L M E}=\rho^{-1}$, it starts with one of the two technological corner solutions (e.g., with $D_{R}=0$ and $\zeta_{R}>0$, or $D_{L}=0$ and $\zeta_{L}>0$ ). In that case, the transitional dynamics are described either by system (A.37a)-(A.37c) or by system (A.41a)-(A.41c).

The end conditions of these dynamical systems depend on whether the transition leads to the fossil steady state, or to the regime with the backstop technology. In the former case, $\theta_{R}$ reaches $\frac{1}{\xi_{R}}$ at time $t=T$. Continuity of the state and co-state variables then implies that the end conditions are $\theta_{R}(T)=\xi_{R}^{-1}, \mu_{L M E}=\rho^{-1}$, and $z(T)=1$. If $\theta_{R}<(>) \xi_{R}^{-1}$, the regime with $D_{R}=0$ and $z<1\left(D_{L}=0\right.$ and $\left.z>1\right)$ applies, because otherwise $z$ would be increasing (decreasing) and never reach unity, as can be seen from combining (A.25e) and (A.25f):

$$
\begin{aligned}
& z \equiv \frac{\lambda_{q R} \xi_{R} Q_{R}}{\lambda_{q L} \xi_{L} Q_{L}} \Rightarrow z \gtreqless 1 \Leftrightarrow \frac{\lambda_{q R} Q_{R}}{\lambda_{q L} Q_{L}} \gtreqless \frac{\xi_{R}^{-1}}{\xi_{L}^{-1}}, \\
& \hat{z}=\frac{\theta_{L}}{\lambda_{q L} Q_{L}}-\frac{\theta_{R}}{\lambda_{q R} Q_{R}} \Rightarrow \hat{z} \gtreqless 0 \Leftrightarrow \frac{\lambda_{q R} Q_{R}}{\lambda_{q L} Q_{L}} \gtreqless \frac{\theta_{R}}{\theta_{L}} .
\end{aligned}
$$

If the transition leads to the regime with the backstop technology, the end conditions are readily obtained by using the continuity of state and co-state variables. First, $\theta_{R}$ converges to $\theta_{H}^{*}$, which it reaches at time $t=T_{H}: \theta_{R}\left(T_{H}\right)=(1-\gamma)^{\sigma} \eta^{\sigma-1}$. Second, (A.25c) and (A.25e) imply that $\mu_{L M E}$ is constant during the backstop regime and equal to $\mu_{L M E}\left(T_{H}\right)=\frac{\xi_{L}}{\rho}$. Finally, (A.45) implies $\lambda_{R}\left(T_{H}\right) Q_{R}\left(T_{H}\right)=0$, yielding $z\left(T_{H}\right)=0$. 


\section{A.2.5 Initial condition}

The initial energy income share in the resource regime can be found by totally differentiating $Z \equiv S Q_{R} /\left(L^{S} Q_{L}\right)$, yielding

$$
\dot{Z}=-\xi_{L}\left(L^{S}-\frac{1-\theta_{R}}{\mu_{L M E}}\right) Z-\frac{1}{L^{S}}\left(\frac{1-\gamma}{\gamma}\right)^{\frac{\sigma}{1-\nu}}\left(\frac{1-\theta_{R}}{\theta_{R}}\right)^{\frac{\nu}{1-\nu}} \frac{1-\theta_{R}}{\mu_{L}}
$$

where we have substituted (A.25a)-(A.25b) and (A.29) into (A.30) to get

$$
\hat{S}=-R / S=-\frac{1}{L^{S}}\left(\frac{1-\gamma}{\gamma}\right)^{\frac{\sigma}{1-\nu}}\left(\frac{1-\theta_{R}}{\theta_{R}}\right)^{\frac{\nu}{1-\nu}} \frac{1-\theta_{R}}{\mu_{L M E}} \frac{1}{Z} .
$$

We define $\mu_{L M E}=h\left(\theta_{R}\right)$ as the fossil equilibrium path in the regime with $\zeta_{R}>0, D_{R}=0$ described in Lemma A.1 with terminal conditions $\theta_{R}=\xi_{R}^{-1}$ and $\mu_{L M E}=\rho^{-1}$ and substitute it into (A.53) to construct the saddle path of the system described by (A.37a) and (A.53) leading to the fossil steady state in $\left(Z, \theta_{R}\right)$-space, which provides $\theta_{R}(0)$ at the fossil path for a given $Z(0)$. Similarly, we define $\mu_{L M E}=k\left(\theta_{R}\right)$ as the backstop equilibrium path in the regime with $\zeta_{R}>0, D_{R}=0$ described in Lemma A.1 with terminal conditions $\theta_{R}=\theta_{H}^{*}$ and $\mu_{L M E}=\xi_{L} / \rho$ and substitute it into (A.53) to construct the saddle path of the system described by (A.37a) and (A.53) in $\left(Z, \theta_{R}\right)$-space with terminal condition $\left(Z, \theta_{R}\right)=\left(0, \theta_{H}^{*}\right)$, which provides $\theta_{R}(0)$ at the backstop path for a given $Z(0)$.

The start condition of the regime with $\zeta_{L}>0, D_{L}=0, \zeta_{R}=0$, and $D_{R}>0$ is immediately obtained from combining (A.25d) and (A.30), yielding

$$
\frac{\theta_{R}(0)}{1-\theta_{R}(0)}=\left(\frac{1-\gamma}{\gamma}\right)^{\sigma}\left(\frac{Q_{R}(0) S(0)}{Q_{L}(0)} \xi_{R}\right)^{\nu-1}
$$

where we have used $\lambda_{q R}(t) Q_{R}(t)=\lambda_{S}(t) S(t)$, which can be seen by defining $V_{R}(t) \equiv e^{-\rho t} \lambda_{q R}(t) Q_{R}(t)$, implying

$$
\dot{V}_{R}(t)=-\lambda_{S}(0) R(t) \Rightarrow V_{R}(t)=V_{R}(\infty)+\lambda_{S}(0) \int_{t}^{\infty} R(\tau) d \tau=\lambda_{S}(0) S(t)
$$

where the first equality uses (A.25a), (A.25f), and (A.25g) and the second equality uses the transversality condition $\lim _{t \rightarrow \infty} V_{R}(t)=0$ (cf. Amigues and Moreaux, 2008, p. 18).

\section{A.2.6 Social planner solution}

Trajectories that satisfy the first-order conditions and the tranversality condition are labeled 'candidate solutions' to the social planner problem. On the basis of our analysis so far, the solution can be stated as in Proposition A.1. 
Proposition A.1 Assuming that $\theta_{R}(0)<\xi_{R}^{-1}$, the following two scenarios can occur:

(i) If $\theta_{H}^{*}<\xi_{R}^{-1}$ the unique solution to the planner problem is a transition to the backstop technology. Throughout the transition, $D_{R}=0$.

(ii) If $\theta_{H}^{*}>\xi_{R}^{-1}$ there are two candidate solutions to the planner problem: a transition to the backstop technology and a transition to the fossil steady state. Along both transitional paths, the $D_{R}=0$ regime occurs. The choice between the two candidate solutions depends on the development of $z(t)$ along the two paths:

(a) If $z(t)<1$ along the entire path towards the backstop technology, both candidate solutions remain.

(b) If $z(t)=1$ for some $t$ along the path towards the backstop technology, the unique solution to the planner problem is a transition to the fossil steady state.

Proof. To prove part (i), let's assume that a transition to the fossil steady state is possible if $\theta_{H}^{*}<$ $\xi_{R}^{-1}$. Note that during the resource regime the inequality (A.27) holds. However, a transition to the fossil steady state implies $\lim _{t \rightarrow \infty} \theta_{R}=\xi_{R}>\theta_{H}^{*}$, so that we get a contradiction. Hence, the transitional path leading to the fossil steady state can be excluded as a candidate solution. The $D_{L}=0$ regime cannot occur, because it follows from $z>1$ that $\xi_{L} / \xi_{R}<\lambda_{R} Q_{R} / \lambda_{L} Q_{L}$. Together with $\theta_{R} / \theta_{L}<\xi_{L} / \xi_{R}$ this implies $\theta_{R} / \theta_{L}<\lambda_{R} Q_{R} / \lambda_{L} Q_{L}$. By using this inequality in combination with (A.25e)-(A.25f), we find $\hat{z}=\theta_{L} / \lambda_{L} Q_{L}-\theta_{R} / \lambda_{R} Q_{R}>0$. Therefore, $\dot{z}>0$, so that $z$ will never reach unity. Hence, the $D_{R}=0$ regime must apply.

To prove part (ii), note that if $\theta_{H}^{*}>\xi_{R}^{-1}$, (A.27) holds during the entire transition to the fossil steady state. Therefore, this path can no longer be excluded as a candidate solution. The argument from part (i) can be used to prove that the $D_{R}=0$ regime must apply along the transitional path to the fossil steady state, along which $\theta_{R}<\xi_{R}^{-1}$. It follows from (A.45) along the transitional path to the backstop technology that $z<1$ just before the switch to the backstop technology, implying that $D_{R}=0$. If $z<1$ throughout the transition to the backstop technology, $D_{R}=0$ from the beginning. However, if, working backwards in time, $z$ reaches unity somewhere at the path, (A.25c)-(A.25f) imply that we are in the fossil steady state with $\xi_{L}=\xi_{R}=0$ and $\theta_{E}=\xi_{R}^{-1}$, where we will stay forever. As a result, the transitional path leading to the introduction of the backstop technology can no longer be a candidate solution. The transition towards the fossil steady state remains as the unique solution to the planner problem. 


\section{A.2.7 Climate change}

The Hamiltonian of the social planner is given by

$$
\mathcal{H}^{C}=\ln C-\psi\left(S_{0}-S\right)+\int_{0}^{1} \lambda_{q L k} \xi_{L} Q_{L} D_{L k} d k+\int_{0}^{1} \lambda_{q R k} \xi_{R} Q_{R} D_{R k} d k-\lambda_{S} R
$$

The corresponding Lagrangian and the first-order conditions are given by, respectively (A.20), (A.21a)-(A.21n). The first-order condition with respect to $S$, (A.21o), becomes

$$
\frac{\partial \mathcal{L}}{\partial S}=\psi=-\dot{\lambda}_{S}+\rho \lambda_{S}
$$

where $\lambda_{S}$ is now to be interpreted as the total cost (i.e., scarcity rent plus climate damage) of resource use. The expressions for the fossil steady state remain unchanged $\left(\theta_{R}=\xi_{R}^{-1}\right.$ and $\mu_{L M E}=\rho^{-1}$ ), but the transitional dynamics are affected by the climate damage. In case of a strictly positive resource scarcity rent, ${ }^{45}$ the differential equation for $\theta_{R}$ in the regime with $D_{L}>0$ and $D_{R}=0$ becomes

$$
\dot{\theta}_{R}=\theta_{R}\left(1-\theta_{R}\right)(1-\nu)\left[\xi_{L} L^{S}-\frac{\psi}{\lambda_{S}}\right]
$$

where we have used (A.25a), (A.25c) and (A.25e) to get $\hat{\mu}_{L M E}=\rho-\xi_{L} L$. Substitution of this result together with $\hat{Q}_{R}=0$, (A.25a), and (A.58) into (A.36) gives (A.59). The differential equations for $\mu_{L M E}, z$, and $Z$ are still given by (A.37b)-(A.37c) and (A.53). Along the transitional path leading to the backstop technology, the scarcity rent may be zero due to the climate damages from atmospheric carbon. ${ }^{46}$ In this case, the solution to differential equation (A.58) reduces to $\lambda_{S}=\psi / \rho$. Accordingly, the differential equation for $\theta_{R}$ in the regime with $D_{L}>0, D_{R}=0$, and $\lambda_{S}=0$ reads

$$
\dot{\theta}_{R}=\theta_{R}\left(1-\theta_{R}\right)(1-\nu)\left[\xi_{L} L^{S}-\rho\right]
$$

A differential equation for $Z$ is no longer needed: the initial condition is now obtained by substitution of (A.25a)-(A.25b) into (A.54), yielding

$$
\frac{1-\theta_{R}(0)}{\theta_{R}(0)}=\left(\frac{\gamma}{1-\gamma}\right)^{\sigma}\left(\frac{\rho}{\psi} \frac{Q_{R}(0)}{Q_{L}(0)} \mu_{L M E}(0)\right)^{1-\nu} .
$$

\footnotetext{
${ }^{45}$ The resource scarcity rent is positive if $\lambda_{S}>\psi / \rho$, where $\psi / \rho$ equals the discounted value of marginal damages of a unit of emitted carbon.

${ }^{46}$ Because the climate cost is constant in utility terms and the backstop cost is constant in output terms, the cost of the backstop relative to the climate damage from resource use in utility terms is declining over time because of output growth. If $S_{0}$ and/or $\psi$ is large enough, the Hotelling rent will drop to zero.
} 
The initial value for $\theta_{R}(0)$ then follows from the point where the line that is implicitly defined by (A.61) intersects the saddle path in $\left(\theta_{R}, \mu_{L M E}\right)$-space.

\section{A.3 Welfare}

The welfare levels associated with time paths that satisfy the necessary conditions are equal to the value of the Hamiltonian corresponding to the two paths at time zero, divided by the pure rate of time preference $\rho$ (cf. Grass et al., 2008, p. 161). Substitution of (7b), and (A.6a)(A.6b) into (A.5) and evaluating at $t=0$, gives the following expression for welfare in a market equilibrium:

$$
\Lambda^{M} \equiv \frac{\mathcal{H}^{M}}{\rho}=\frac{1}{\rho}\left[\ln C^{M}(0)+\frac{\mu_{L}(0)}{1-(1-\beta)^{2}}\left(\frac{\rho}{L^{S}}+1\right)-1\right]
$$

where the initial consumption level can be found by combining (1), (2a)-(2b), (4), (8), and (16) to get

$$
C^{M}(0)=\frac{Q_{L}(0)}{\mu_{L}(0)} L^{S} \gamma^{\frac{\sigma}{\nu-1}}\left[1-(1-\beta)^{2}\right] \beta(1-\beta)^{2 \frac{1-\beta}{\beta}}\left(1-\theta_{R}(0)\right)^{\frac{1}{1-\nu}}
$$

Similarly, the expression for welfare along time paths that satisfy the necessary conditions of the social optimum can be found by substituting (A.29), and (A.25a)-(A.25c) into (A.19), and evaluating at $t=0$, yielding

$$
\Lambda^{S} \equiv \frac{\mathcal{H}^{S}}{\rho}=\frac{1}{\rho}\left[\ln \left(C^{S}(0)\right)+\mu_{L M E}(0) L^{S}-1\right]
$$

where the initial consumption level follows from (1), (4), (A.21c)-(A.21d), (A.21f), (A.25a), (A.28), and (A.29):

$$
C^{S}(0)=\gamma^{\frac{\sigma}{\nu-1}} \beta(1-\beta)^{\frac{1-\beta}{\beta}} \frac{Q_{L}(0)}{\mu_{L M E}(0)}\left(1-\theta_{R}(0)\right)^{\frac{1}{1-\nu}}
$$

To derive the welfare difference measure that we use in Figures 10 and 12, we first need the following welfare levels:

$$
\begin{aligned}
& \text { (A.66a) } \Lambda_{H}^{i}=\int_{0}^{\infty} \ln \left(C_{H}^{i}(0) e^{\int_{0}^{t} \hat{C}_{H}^{i}(s) d s}\right) e^{-\rho t} d t=\frac{\ln C_{H}^{i}(0)}{\rho}+e^{-\rho t} \int_{0}^{\infty} \int_{0}^{t} \hat{C}_{H}^{i}(s) d s d t, \\
& \text { (A.66b) } \Lambda_{F}^{i}=\int_{0}^{\infty} \ln \left(C_{F}^{i}(0) e^{\int_{0}^{t} \hat{C}_{F}^{i}(s) d s}\right) e^{-\rho t} d t=\frac{\ln C_{F}^{i}(0)}{\rho}+e^{-\rho t} \int_{0}^{\infty} \int_{0}^{t} \hat{C}_{F}^{i}(s) d s d t .
\end{aligned}
$$


where $i=M, S$, and the subscripts $H$ and $F$ indicate the backstop and the fossil transitional path, respectively. Our measure $\Delta^{i}$ now follows from the equality

$$
\Lambda_{F}^{i}=\frac{\ln \left[\left(1+\Delta^{i}\right) C_{H}^{i}(0)\right]}{\rho}+e^{-\rho t} \int_{0}^{\infty} \int_{0}^{t} \hat{C}_{H}^{i}(s) d s d t
$$

Combining (A.66a) and (A.67), we get

$$
\Lambda_{F}^{i}=\frac{\ln \left(1+\Delta^{i}\right)}{\rho}+\Lambda_{H}^{i} \Leftrightarrow \Delta^{i}=e^{-\rho\left(\Lambda_{H}^{i}-\Lambda_{F}^{i}\right)}-1
$$

\section{A.4 Relaxation algorithm}

For the numerical analysis of the transitional dynamics of the model, we have used the relaxation algorithm described by Trimborn et al. (2008). The essence of the algorithm is to replace a system of $N$ differential equations by a system of approximate finite-difference equations on a mesh of $M$ points in time, and to solve the latter system with a Newton-type iteration process. Details can be found in Trimborn et al. (2008). In the remainder of this section we describe the numerical procedure that we have used to construct the different transition paths in our model economy.

To determine the transition path leading to the implementation of the backstop technology in the market economy, we work backwards in time. First, we numerically solve the fossil regime with purely labor-augmenting technical change and use $\theta_{R}=(1-\gamma)^{\sigma} \eta^{\sigma-1}, \mu_{L}=$ $\xi_{L} \beta(2-\beta) L^{S} /\left(\xi_{L} L^{S}+\rho\right), Z=0$, and $\chi=0$ as end conditions. Then, we find the point on the numerically determined transition path where $\chi=1$, to identify the the possible start point of this regime. However, if $Z$ drops below $Z(0)$ during this regime, we use the point on the determined equilibrium path where $Z$ equals $Z(0)$ as the actual start point of the transition path. If not, there must be a preceding fossil regime with both types of technical change, the end condition of which is given by the the vector $\left(\theta_{R}, \mu_{L}, Z, \chi\right)$ on the determined equilibrium path where $\chi=1$. Then, we use the relaxation algorithm to find the transition path in this regime and find the point where $D_{R}=0\left(D_{R}\right.$ can be expressed in terms of $\theta_{R}$ and $\left.\mu_{L}\right)$ to identify the the possible start point.

If $Z$ drops below $Z(0)$ during this regime, we use the point on the determined equilibrium path in the regime with both types of technical change where $Z$ equals $Z(0)$ as the actual start point of the transition path. If not, there must be a preceding fossil regime with purely labor-augmenting technical change, the end condition of which is given by the vector $\left(\theta_{R}, \mu_{L}, Z, \chi\right)$ on the determined equilibrium path corresponding to the point where $D_{R}=0$. Subsequently, we use the relaxation algorithm once more to find the transition path in this regime. Finally, we use the point on the determined equilibrium path where $Z$ equals $Z(0)$ 
as the start point of the equilibrium path that leads to the implementation of the backstop technology. To determine the transition path in the market economy leading to the fossil steady state, or to determine the transition paths in the social optimum, we use a similar procedure. 


\section{References}

Acemoglu, D., "Why Do New Technologies Complement Skills? Directed Technical Change And Wage Inequality," The Quarterly Journal of Economics 113 (November 1998), 1055-1089.

__, "Directed Technical Change," Review of Economic Studies 69 (October 2002), 781-809.

_ - "Labor- And Capital-Augmenting Technical Change," Journal of the European Economic Association 1 (03 2003), 1-37.

Acemoglu, D., P. Aghion, L. Bursztyn and D. Hemous, "The Environment and Directed Technical Change," American Economic Review 102 (February 2012), 131-66.

Amigues, J.-P. And M. Moreaux, "Dedicated Technical Progress with a Non-renewable Resource: Efficiency and Optimality," IDEI Working Papers 497, Institut d'conomie Industrielle (IDEI), Toulouse, January 2008.

ANDRÉ, F. J. AND S. SMULDERS, "Fueling growth when oil peaks: Directed technological change and the limits to efficiency," European Economic Review 69 (2014), 18 - 39.

Arthur, W. B., "Competing Technologies, Increasing Returns, and Lock-In by Historical Events," The Economic Journal 99 (1989), pp. 116-131.

Benchekroun, H. And C. Withagen, "The optimal depletion of exhaustible resources: A complete characterization," Resource and Energy Economics 33 (September 2011), 612-636.

Benhabib, J. AND R. E. FARMER, "Indeterminacy and Increasing Returns," Journal of Economic Theory 63 (1994), $19-41$.

Benhabib, J. AND R. Perli, "Uniqueness and Indeterminacy: On the Dynamics of Endogenous Growth," Journal of Economic Theory 63 (June 1994), 113-142.

Chamley, C., "Externalities and Dynamics in Models of "Learning or Doing"," International Economic Review 34 (August 1993), 583-609.

Cheikbossian, G. AND F. Ricci, "Resource Depletion and Directed R\&D: Expectations-Driven Equilibria," mimeo, 2013.

Cooper, R. And A. John, "Coordinating Coordination Failures in Keynesian Models," The Quarterly Journal of Economics 103 (August 1988), 441-63.

CozzI, G., "Animal spirits and the composition of innovation," European Economic Review 49 (April 2005), 627-637. 
- "Self-fulfilling prophecies in the quality ladders economy," Journal of Development Economics 84 (September 2007), 445-464.

Dasgupta, P. And G. Heal, "The Optimal Depletion of Exhaustible Resources," The Review of Economic Studies 41 (1974), 3-28.

DAVID, P. A., "Clio and the Economics of QWERTY," American Economic Review 75 (May 1985), 332-37.

Di MARIA, C. AND S. VAlente, "Hicks meets Hotelling: the direction of technical change in capitalresource economies," Environment and Development Economics 13 (December 2008), $691-717$.

Edenhofer, O. And M. Kalkuhl, "Das grüne Paradoxon Menetekel oder Prognose," in J. M. H. N. F. Beckenbach, J. Weimann and U. Witt, eds., Jahrbuch Ökologische Ökonomik, Band 6: Diskurs Klimapolitik (Metropolis, Marburg, 2009), 115-121.

GolLIN, D., "Getting Income Shares Right," Journal of Political Economy 110 (April 2002), 458474.

Grass, D., J. P. Caulkins, G. Feichtinger, G. Tragler and D. A. Behrens, Optimal Control of Nonlinear Processes With Applications in Drugs, Corruption, and Terror (Springer-Verlag, Berlin Heidelberg, 2008).

Grossman, G. M. And E. HelPman, Innovation and Growth in the Global Economy, volume 1 of MIT Press Books (The MIT Press, 1991).

HART, R., "Directed technological change: It's all about knowledge," Working Paper Series 2012:02, Department Economics, Swedish University of Agricultural Sciences, May 2012.

__ "Directed technological change and factor shares," Economics Letters 119 (2013), 7780.

Hicks, J., The Theory of Wages (London: MacMillan, 1932).

HowitT, P., "Steady Endogenous Growth with Population and R \& D Inputs Growing," Journal of Political Economy 107 (August 1999), 715-730.

Jones, C. I., "R\&D-Based Models of Economic Growth," Journal of Political Economy 103 (August 1995), 759-84.

Kiley, M. T., "The Supply of Skilled Labour and Skill-Biased Technological Progress," Economic Journal 109 (October 1999), 708-24. 
Koetse, M. J., H. L. De Groot and R. J. FloraX, "Capital-energy substitution and shifts in factor demand: A meta-analysis," Energy Economics 30 (September 2008), 2236-2251.

Krugman, P., "History Versus Expectations," The Quarterly Journal of Economics 106 (1991), pp. 651-667.

LUCAS, R. J., "On the mechanics of economic development," Journal of Monetary Economics 22 (July 1988), 3-42.

MATSUYAMA, K., "Increasing Returns, Industrialization, and Indeterminacy of Equilibrium," The Quarterly Journal of Economics 106 (1991), 617-650.

Murphy, K. M., A. Shleifer And R. W. Vishny, "Industrialization and the Big Push," Journal of Political Economy 97 (October 1989), 1003-26.

Nordhaus, W. D., "The Allocation of Energy Resources," Brookings Papers on Economic Activity 4 (1973), 529-576.

Pittel, K. And L. Bretschger, "Sectoral Heterogeneity, Resoure Depletion, and Directed Technical Change: Theory and Policy," Technical Report, 2010.

Romer, P. M., "Increasing Returns and Long-run Growth," Journal of Political Economy 94 (October 1986), 1002-37.

__ "Endogenous Technological Change," Journal of Political Economy 98 (October 1990), S71-102.

SCHMIDT, R. C. AND R. MARSCHINSKI, "A model of technological breakthrough in the renewable energy sector," Ecological Economics 69 (December 2009), 435-444.

SinN, H.-W., "Public policies against global warming: a supply side approach," International Tax and Public Finance 15 (August 2008), 360-394.

SMUlders, S. AND M. DE NoOIJ, "The impact of energy conservation on technology and economic growth," Resource and Energy Economics 25 (February 2003), 59-79.

Solow, R. M., "The Economics of Resources or the Resources of Economics," The American Economic Review 64 (1974a), 1-14.

—_, "Intergenerational Equity and Exhaustible Resources," The Review of Economic Studies 41 (1974b), 29-45.

Stiglitz, J., "Growth with Exhaustible Natural Resources: Efficient and Optimal Growth Paths," The Review of Economic Studies 41 (1974a), 123-137. 
Stiglitz, J. E., "Growth with Exhaustible Natural Resources: The Competitive Economy," The Review of Economic Studies 41 (1974b), 139-152.

The Conference BoArd, Total Economy Database (New York, 2011).

Trimborn, T., K.-J. Koch And T. M. Steger, "Multidimensional Transitional Dynamics: A Simple Numerical Procedure," Macroeconomic Dynamics 12 (June 2008), 301-319.

UnRuH, G. C., "Understanding carbon lock-in," Energy Policy 28 (October 2000), 817-830.

U.S. Energy Information Administration, Annual Energy Review (Washington D.C., 2011).

UzawA, H., "Optimum Technical Change in An Aggregative Model of Economic Growth," International Economic Review 6 (1965), 18-31.

VAn Der MeiJden, G., The Macroeconomic Dynamics of Trade Liberalization, Resource Exploitation, and Backstop Technologies (Ph.D. Dissertation, Tilburg University, 2013).

Van der Ploeg, F. And C. Withagen, "Growth, Renewables, And The Optimal Carbon Tax," International Economic Review 55 (02 2014), 283-311.

— - "Global Warming and the Green Paradox: A review of Adverse Effects of Climate Policies," Review of Environmental Economics and Policy forthcoming (2015).

VAN DER WERF, E., "Production functions for climate policy modeling: An empirical analysis," Energy Economics 30 (November 2008), 2964-2979.

WORLD BANK, World Development Indicators (Washington, D.C.: World Bank, 2014). 